\title{
Diagnosis of Localized Faults in Multistage Gearboxes: A Vibrational Approach by Means of Automatic EMD-Based Algorithm
}

\author{
M. Buzzoni, E. Mucchi, G. D'Elia, and G. Dalpiaz \\ Engineering Department, University of Ferrara, Via Saragat, 1 I-44122 Ferrara, Italy \\ Correspondence should be addressed to E. Mucchi; emiliano.mucchi@unife.it
}

Received 26 July 2017; Accepted 14 September 2017; Published 30 October 2017

Academic Editor: Rafał Burdzik

Copyright (c) 2017 M. Buzzoni et al. This is an open access article distributed under the Creative Commons Attribution License, which permits unrestricted use, distribution, and reproduction in any medium, provided the original work is properly cited.

\begin{abstract}
The gear fault diagnosis on multistage gearboxes by vibration analysis is a challenging task due to the complexity of the vibration signal. The localization of the gear fault occurring in a wheel located in the intermediate shaft can be particularly complex due to the superposition of the vibration signature of the synchronous wheels. Indeed, the gear fault detection is commonly restricted to the identification of the stage containing the faulty gear rather than the faulty gear itself. In this context, the paper advances a methodology which combines the Empirical Mode Decomposition and the Time Synchronous Average in order to separate the vibration signals of the synchronous gears mounted on the same shaft. The physical meaningful modes are selected by means of a criterion based on Pearson's coefficients and the fault detection is performed by dedicated condition indicators. The proposed method is validated taking into account simulated vibrations signals and real ones.
\end{abstract}

\section{Introduction}

Multistage gearboxes are employed in a wide range of mechanical systems and represent crucial components for the correct functioning of the entire machine. Since they are often subjected to faults due to manufacturing errors or heavy working conditions, the gear fault identification is of prime importance in order to reduce the maintenance costs as well as to restrict machine downtimes. In this context, the exact knowledge of the fault position by means of nondestructive techniques simplifies the maintenance process avoiding burdensome visual inspections.

Vibration-based diagnosis represents an effective approach for the gear fault diagnosis [1]. In the last decades, many researches have been focused on the development and testing of signal processing techniques for the identification of localized gear faults. The success of the fault identification strongly depends on the employed signal processing techniques, the system typology under investigation, and the working condition. In fact, the state of the art about the identification of localized gear faults covers a wide range of different approaches such as the following: the cyclostationary theory [2-4], which takes advantage of the hidden periods embodied in the vibration signals; the Kurtogram [5] for the selection of the frequency band associated with the maximum Spectral Kurtosis; time-frequency signal representations like Continuous Wavelet Transform [6]; the blind deconvolution algorithms [7, 8], which estimate the excitation source due to the presence of the fault from the noisy observation; condition indicators based on the Time Synchronous Average [9].

Unfortunately, the aforementioned approaches allow for identifying the rotation period of the shaft synchronous with the faulty gear rather than the faulty gear itself. Hence, the exact identification of the faulty gear is not a trivial task if two or more gears are installed in the same shaft (which is very common in multistage gearboxes). As the authors are aware, no works can be found in the specialized literature dealing with such a tricky problem. Hence, encouraged by this lack, the present research is focused on the investigation of this issue having both industrial and academic interest. 
In this work, the signal separation is based on the EMD (Empirical Mode Decomposition) which represents a fascinating approach in the field of time-frequency signal processing techniques. The EMD was introduced for the first time by Huang et al. [10] and it is a self-adaptive time-frequency analysis technique. The EMD decomposes the original signal into a set of oscillatory modes (called also Intrinsic Mode Functions) on the basis of the local time scales of the signal rather than on a predetermined kernel, as in the case of the Continuous Wavelet Transform. The EMD is effective for the analysis of signals that exhibit nonstationary and nonlinear behavior. Since the EMD is fully data-driven and adapted for the analysis of nonstationary signals, it is particularly suitable for the goal of this paper. Many efforts have been made in order to improve the effectiveness of the EMD algorithm, restricting its intrinsic drawbacks [11, 12]. The EEMD (Ensemble Empirical Mode Decomposition) [13], the CEEMD (Complementary Ensemble Empirical Mode Decomposition) [14], and the CEEMDAN (Complete Ensemble Empirical Mode Decomposition with Adaptive Noise) [15] are among the most popular improved EMD methods proposed in the literature.

EMD has been successfully used in a number of different research fields (speech recognition, chemistry, biology, medicine, etc.) but only in the last decade has EMD been exploited also for the identification of the gear faults, as gear cracks $[16,17]$, broken teeth [18], or wear [19]. In this context, Lin and Chen [20] exploited the EEMD for the extraction of multiple fault information from the vibration signals measured on gearboxes, a diagnostic method for wind turbine planetary gearboxes based on the EEMD has been proposed by Feng et al. [21] and the CEEMD combined with Permutation Entropy has been used for the identification and the severity recognition of gear faults by Zhao et al. [22]. On the other hand, no researches can be found in the literature about the gear fault diagnostic by means of CEEMDAN, even if its effectiveness has been demonstrated in other applications as the bearing fault identification [23, 24]. A complete literature review about the use of EMD for rotating machine diagnostics can be found in [25].

On this basis, the proposed work aims at developing a EMD-based methodology for the identification of the faulty wheel in multistage gearboxes, in the case of synchronous wheels mounted on intermediate shafts. Specifically, this method allows for the precise detection of the faulty gear rather than the faulty stage. In fact, the faulty gear detection is a limitation of the traditional signal processing techniques when a fault occurs in an intermediate stage. As mentioned before, this research tries to fill the gap in the specialized literature by facing this challenging case, being also of particular concern in many industrial applications. In this regard, care has been taken on the validation of the algorithm as well as on the reduction of the user interactions. For this purpose, selection criterion of the oscillatory modes estimated by a EMD-based algorithm have been advanced, in order to separate the Time Synchronous Average of the vibration signal into two representative vibration signals of the investigated wheels. Different EMD algorithms are taken into account (EMD, EEMD, and CEEMDAN) in order to verify how the signal separation is influenced. The localized fault identification has been quantified by means of dedicated statistical indicators that reflect the gear condition. The method is validated taking into account both simulated signals and real vibration signals.

The paper is structured as follows: Section 2 outlines the theoretical background; Section 3 introduces the problem statement and the description of the method; the methodology has been tested using simulated signals in Section 4; Section 5 focuses on the validation by means of real vibration signals; final remarks are drawn in Section 6.

\section{Theoretical Background}

In this section, the main signal processing tools necessary for the comprehension of the proposed methodology are concisely introduced. Only the fundamental concepts are described avoiding unnecessary theoretical explanations.

2.1. The Time Synchronous Average. The vibration signals acquired on gearboxes can be considered as wide-sense cyclostationary signals [26]. The first-order cyclostationary part is particularly significant for the gear fault identification and the Time Synchronous Average (TSA) is a common estimator of such a cyclostationary quantity [3, 27]. In general, the TSA can be considered as the ensemble average of the vibration signal synchronized with a certain rotating component having rotation period $T$. Commonly, the TSA is typically performed into the angle domain rather than the time domain. In fact, the cyclostationarity on mechanical systems follows the periodicity imposed by the kinematics of the system, which is locked in the angle variable. Thus, many mechanical systems exhibit cyclostationarity with respect to rotation rather than to time.

Let $x(\theta)$ be the vibration signal synchronized with rotation $\theta$ of a certain rotating mechanical component taken as reference. Considering a periodicity of $\Theta=2 \pi$ and an integer number $N$ of revolutions, the length of $x(\theta)$ is $N \Theta$. Thus, the Time Synchronous Average, $x_{\mathrm{TSA}}(\theta)$, of $x(\theta)$ can be defined as

$$
x_{\mathrm{TSA}}(\theta)=\frac{1}{N} \sum_{n=0}^{N-1} x(\theta+n \Theta) \quad \text { with } 0 \leq \theta<2 \pi .
$$

The change of variable from time to angle implies that the frequency variable will change accordingly. The new frequency variable is called "order" and it is defined as the ratio between cycles and machine speed. Taking into account a proper number of averages, the main result of the TSA is the strong attenuation of all the nonperiodic components with respect to the reference and the improvement of the SNR (Signal-to-Noise Ratio). Furthermore, the angle domain TSA can strongly reduce the effects of the speed variation that mask the effects due to possible faults. Hence, this signal processing technique is particularly effective with noisy signals that embody a number of components having different periods, as the multistage gearboxes.

2.2. The Empirical Mode Decomposition. The EMD is a selfadaptive signal decomposition technique that separates the 
signal in several oscillatory components called IMF (Intrinsic Mode Function) or just modes. Each IMF has to meet two properties: (i) the zero-crossing points and the extrema have to be equal or differ by one; (ii) at any point the mean value of the envelope evaluated by the local maxima and by the local minima has to be zero [10]. By virtue of these properties, the last component extracted by the EMD is a monotonic signal, called residue. As the name suggests, there is still a lack of a general theory about EMD. This pitfall has been investigated by some authors $[28,29]$ in the attempt to formulate a theoretical foundation of the method, but it is still an open question.

For the sake of brevity, the essential steps of the EMD algorithm are described in Figure 1, but a more comprehensive explanation about this algorithm can be found in [10]. The envelope process and the sifting process are the key points of the algorithm in Figure 1. The sifting process is a recursive procedure which ensures that the estimated modes can satisfy the characteristic properties of the IMF; the envelope process, instead, is the evaluation of envelopes of maxima and minima of the signal.

The main drawbacks of the EMD are the mode mixing and the end effect. The mode mixing concerns the combination of signals with widely different scales, while the end effect is the signal distortion at the extremity of the signal itself. These shortcomings can undermine the physical meaning of the estimated IMFs. In order to enhance the results of the EMD, several improved versions of the EMD algorithm have been developed in the last 20 years [13-15].

2.3. The Ensemble Empirical Mode Decomposition. The EEMD (Ensemble Empirical Mode Decomposition) [30] is an improved version of the EMD and its algorithm is summarized in Figure 2. Unlike the EMD, which could estimate IMFs affected by severe mode mixing, the EEMD calculates the so-called true modes or IMF* and represents a more reliable decomposition of the signal.

Departing from original signal $x(t)$, a new set of $N$ signals (where $N$ represents the number of trials or realizations) is created by adding different zero-mean white noise $w(t)$ of finite variance $\sigma$. Then, the EMD is performed for each signal $x_{n}$ obtaining $N$ mode sets composed of $K$ modes each. At the end, the true IMF set composed of a number $K$ of true modes IMF $^{*}$ is evaluated by ensemble averaging each $k$ th IMF set previously obtained.

Different from the EMD, the EEMD depends on two arbitrary parameters: the number of trials and the variance of the added white noise. The relationship between these parameters is as follows:

$$
\varepsilon=\frac{\sigma}{\sqrt{N}},
$$

where $N$ is the number of trials, $\sigma$ is the variance of the added noise, and $\varepsilon$ is the error standard deviation defined as the difference between the original signal and the corresponding IMFs. The proper selection of these parameters is needed in order to obtain negligible errors. Commonly [10, 31], a few hundreds of averages and $\sigma \approx 0.02$ are usually enough in order to obtain satisfactory results. However, the selection of $\sigma$ depends on the application since high values of $\sigma$ are suitable for data dominated by low-frequency signals and vice versa [10].

\subsection{The Complete Ensemble Empirical Mode Decomposition} with Adaptive Noise. The Complete Ensemble Empirical Mode Decomposition with Adaptive Noise (CEEMDAN) represents a step further with respect to EEMD and it has been proposed for the first time by Torres et al. [15]. As shown in Figure 2, the EEMD calculates the true modes averaging a certain number of noisy IMF sets evaluated independently of each other and each $\mathrm{IMF}_{n, k}$ is determined considering the residue of corresponding previous mode $\mathrm{IMF}_{n, k-1}$.

On the contrary, the CEEMDAN algorithm (Figure 3) does not estimate the true modes in a single step as in the EEMD but such true modes are calculated sequentially. Specifically, for the estimation of each true mode, the CEEMDAN algorithm takes into account the contribution of the residue evaluated from the previous true mode. Therefore, the CEEMDAN guarantees the exact correspondence between the original signal and the set of decomposed signals, which is not ensured by the EEMD [32]:

$$
x(t)=\sum_{n=k}^{K} \operatorname{IMF}_{k}^{*}(t)+r(t) .
$$

Referring to Figure 3, at each iteration, the $k$ th true mode is estimated from the previous residue calculated by the $(k-$ 1)th mode perturbed by the white noise. Otherwise, in this algorithm the added white noise for the estimation of the $k$ th IMF actually is the $k$ th mode obtained performing the EMD on the white noise. A more exhaustive explanation of the CEEMDAN algorithm can be found in $[32,33]$. The main advantages of this method are the exact reconstruction of the signal and the possibility of changing the noise level at each stage.

2.5. Gear Fault Identification by Condition Indicators. The presence of gear faults leads to changes in the vibration signature measured on the gearboxes. A number of researches can be found in the literature about the development of parameters for the quantification of the vibration signature modification $[4,9]$. These parameters are generally called condition indicators (CIs).

In this study, the following standard CIs have been considered: kurtosis, Crest Factor (CF), and FM0. The kurtosis is the standardized moment of a probability distribution, the $\mathrm{CF}$ is the ratio between the peak value and the RMS (Root Mean Square) value and the FM0 is the ratio between the peak-topeak value and the sum of the gear mesh harmonics. These parameters are particularly effective for the identification of local changes in the vibration signature, as in the case of localized gear faults.

Furthermore, two new CIs are proposed based on the vibration signal RMS values evaluated for each tooth: Crest Pitch Factor (CPF) and the Normalized Skewness Variance Product (NSVP). In other words, the angle domain vibration 


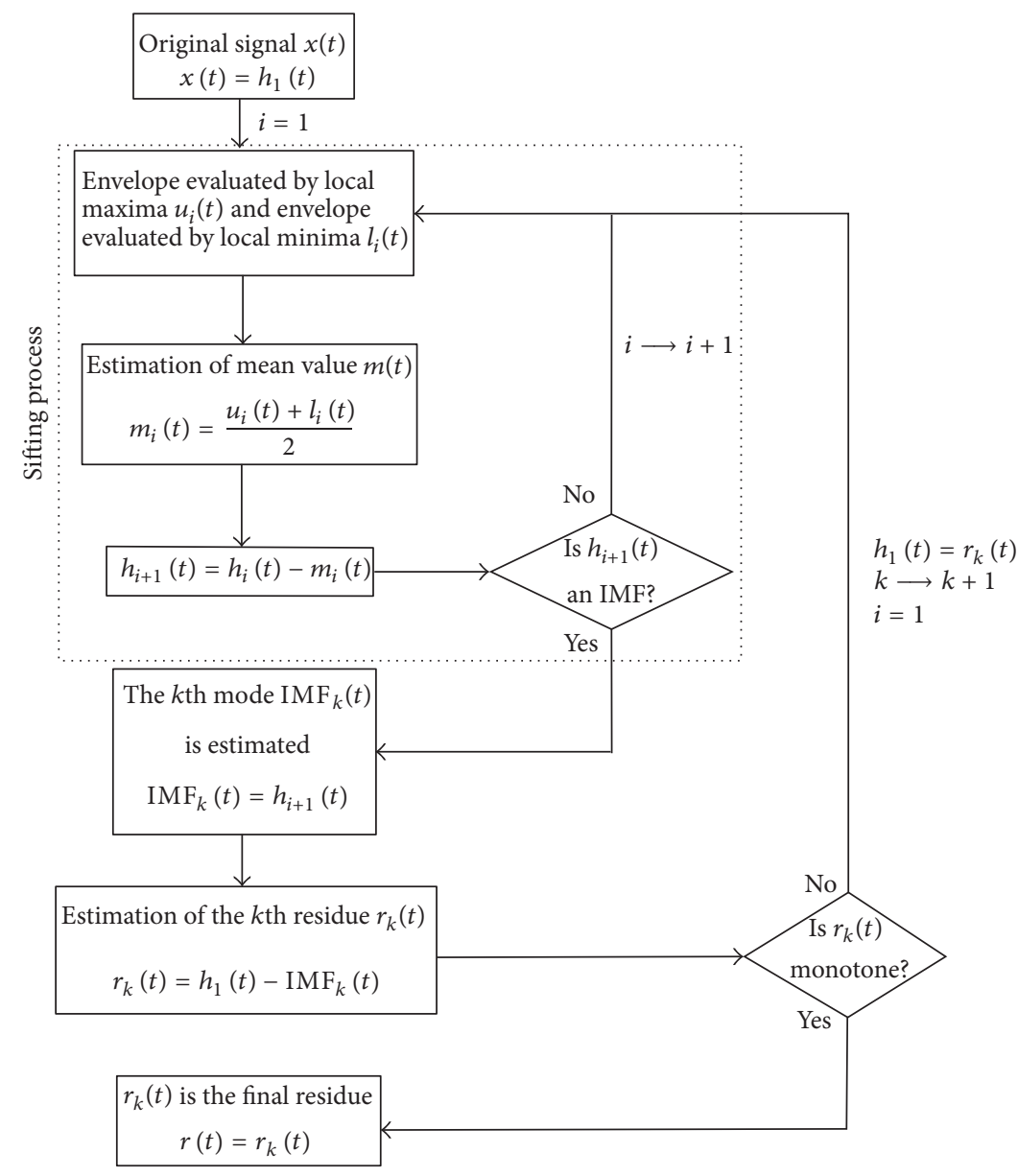

FIgURE 1: Flow-chart of the EMD algorithm.

signal referred to a single revolution (namely, $x(\theta)$ ) is split into a number of parts equal to the number of teeth; then, the RMS value is estimated for each part. Let $\mathrm{RMS}_{p}{ }^{i}$ be the RMS value of the $i$ th tooth, and $\mathrm{RMS}_{p}{ }^{i}$ is defined as follows:

$$
\begin{array}{r}
\operatorname{RMS}_{p}{ }^{i}=\operatorname{RMS}\left[x\left(\theta_{p}+\frac{z}{2 \pi}(i-1)\right)\right] \\
\text { with } 0 \leq \theta_{p}<\frac{z}{2 \pi},
\end{array}
$$

where $\theta_{p}$ is the angular pitch, $z$ is the number of teeth, and RMS is the Root Mean Square operator. The whole set of $\mathrm{RMS}_{p}{ }^{i}$ is

$$
\left\{\mathrm{RMS}_{p}\right\}=\left\{\begin{array}{c}
\mathrm{RMS}_{p}{ }^{1} \\
\vdots \\
\mathrm{RMS}_{p}{ }^{i} \\
\vdots \\
\mathrm{RMS}_{p}{ }^{z}
\end{array}\right\}
$$

For a gear having localized faults, a local deviation from the mean value of $\mathrm{RMS}_{p}$ is expected. The first CI, called CPF, is defined as the ratio of the maximum value of $\mathrm{RMS}_{p}$ with respect to the $\mathrm{RMS}_{p}$ ensemble mean value:

$$
\mathrm{CPF}=\frac{\max \left[\mathrm{RMS}_{p}\right]}{E\left[\mathrm{RMS}_{p}\right]},
$$

where $E$ is the ensemble mean operator. Now, let us consider the difference among adjacent $\mathrm{RMS}_{p}^{i}$ values:

$$
\left\{\mathrm{RMS}_{p d}\right\}=\left\{\begin{array}{c}
\mathrm{RMS}_{p}{ }^{2}-\mathrm{RMS}_{p}{ }^{1} \\
\vdots \\
\mathrm{RMS}_{p}{ }^{i}-\mathrm{RMS}_{p}{ }^{i-1} \\
\vdots \\
\mathrm{RMS}_{p}{ }^{z}-\mathrm{RMS}_{p}{ }^{z-1}
\end{array}\right\} .
$$

Intuitively, $\mathrm{RMS}_{p d}$ values should be close to zero for healthy gears since the variation between two consecutive RMS pitch values is slight. On the other hand, $\mathrm{RMS}_{p d}$ exhibits nonzero values when local changes of the vibration signature occur, since the vibration signature of a healthy meshing tooth is different from a faulty one. On the basis of these 


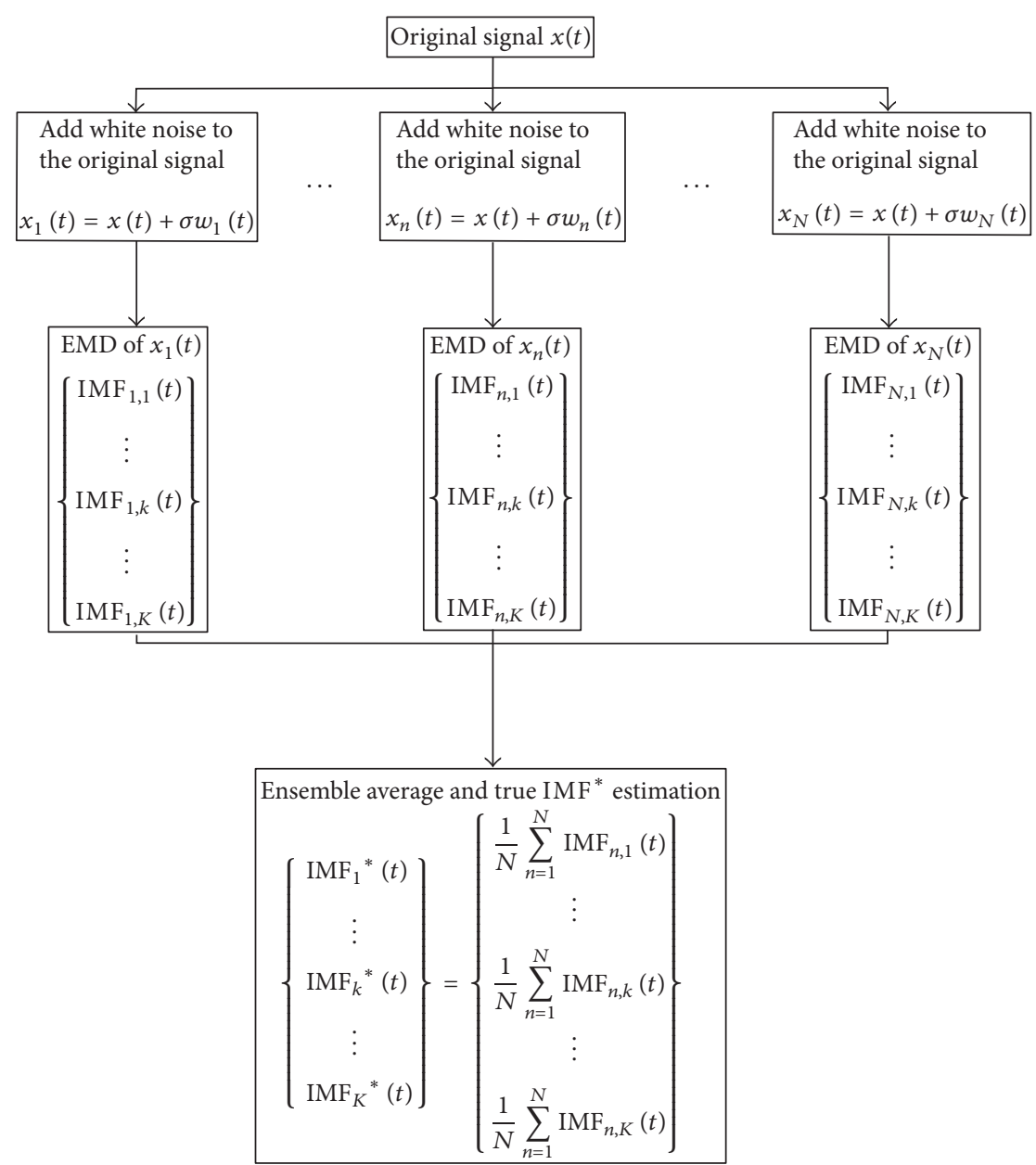

FIgURE 2: Flow-chart of the EEMD algorithm with $N$ trials and $K$ modes per trial.

considerations, the second proposed CI, called NSVP, is defined as the product between skewness and variance of the $\mathrm{RMS}_{p d}$ normalized by the peak-to-peak value of $\mathrm{RMS}_{p d}$ :

$$
\mathrm{NSVP}=\frac{\operatorname{var}\left[\mathrm{RMS}_{p d}\right] \text { skew }\left[\mathrm{RMS}_{p d}\right]}{\mathrm{pp}\left[\mathrm{RMS}_{p d}\right]},
$$

where var is the variance operator, skew is the skewness operator, and $\mathrm{pp}$ is the peak-to-peak value.

\section{Problem Statement and the Proposed Method}

In theory, the vibration signal of a gearbox operating at steady-state condition appears as a composition of harmonics having as fundamental frequencies the meshing frequencies. The localized gear faults can appear in the vibration signals as impulsive components and local modulation of amplitude and/or phase [34]. These local components are visible on the spectrum as side-bands centred on the meshing frequencies. The pure impulsive component depends on the period of the occurrence (the shaft period) and on the impulse response function of the system, and thus it depends on parameters that are not strictly related to gear II or gear III. The local modulation of amplitude and phase is a function of the gear mesh frequency of the faulty wheel. Thus, considering two gears with different (and not multiple) number of teeth, the analysis of the local modulation period due to the localized defect should lead to the identification of the faulty gear. Indeed, the modulation depends on the gear mesh frequency that is different for the considered gears.

When the TSA is performed according to a certain shaft period, it is possible to set apart only the tones that are synchronous with the shaft of interest. However, the TSA cannot separate those gear mesh harmonics belonging to two or more gears which are rotating in the same shaft (which is a very common case in multistage gearbox). Hence, in order to identify the faulty gear, the basic idea of this work is to exploit the local change of the meshing vibration due to the amplitude and phase modulation rather than the impulsive component. A signal separation method based on EMD algorithm is described hereafter in order to overcome this problem, which is fairly common in practical applications 


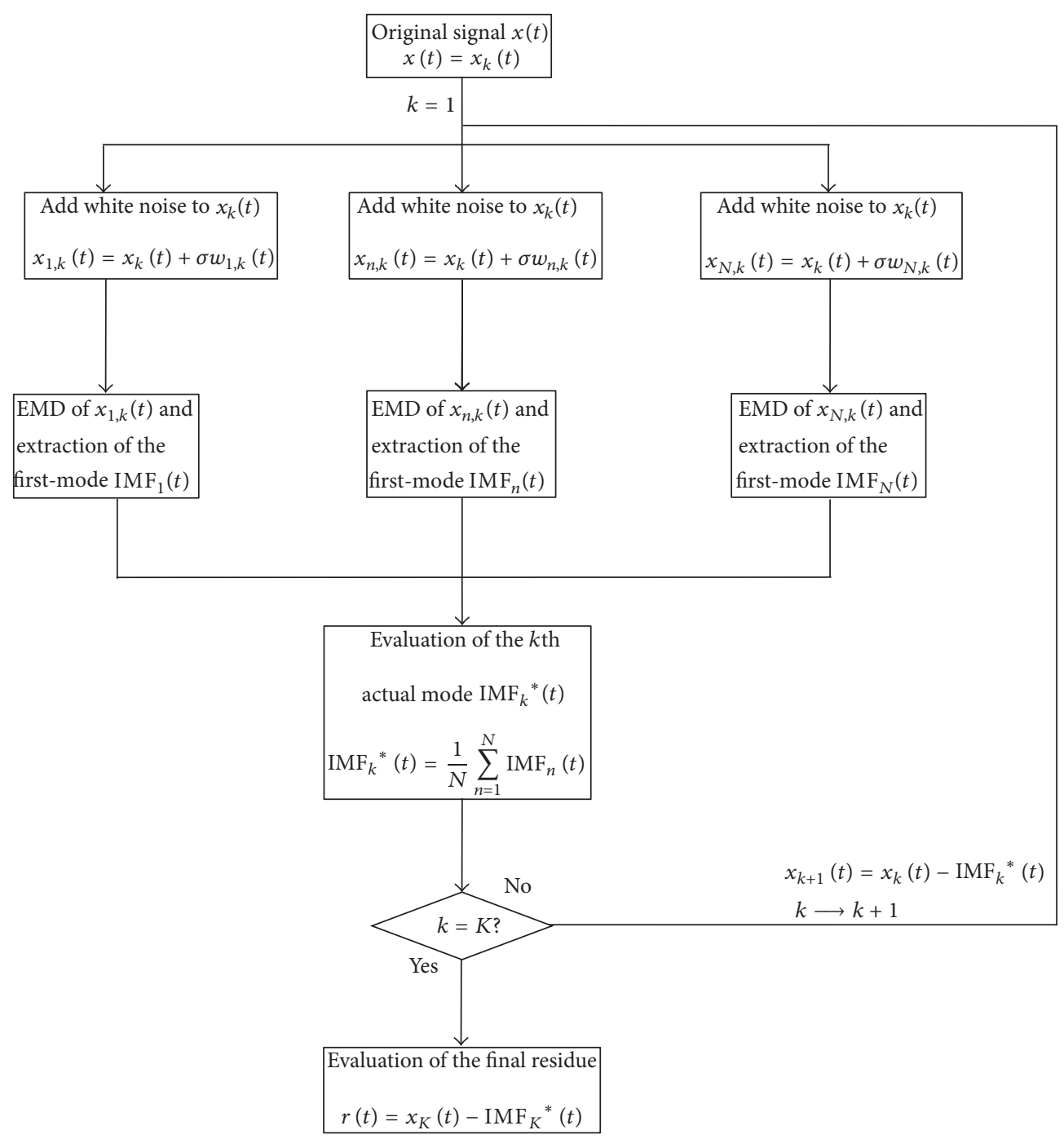

FIGURE 3: Flow-chart of the CEEMDAN algorithm.

with a significant implication concerning the reduction of maintenance costs and time.

Let us consider the two-stage gearbox shown in Figure 4, composed of four spur gears (namely, I, II, III, and IV) operating at steady-state conditions. Furthermore, let us suppose the presence of a localized fault on wheel II.

Thus, taking advantage from the EMD-based decomposition, two representative vibration signatures characterizing the meshing vibration of gear II and gear III, respectively, can be built taking into account the physically meaningful IMFs. The physically meaningful IMFs are intended as those IMFs that describe the gear mesh vibration signature of the gear of interest. Therefore, the representing signal of the gear will be the sum of these modes (if they are more than one). Figure 5 describes the proposed methodology (summarized in 4 fundamental steps) for the generic two-stage gearbox in Figure 4.

Under the assumption that the gear fault on the intermediate shaft is evident on the TSA, the first step involves the low-pass filtering since several high frequency signal

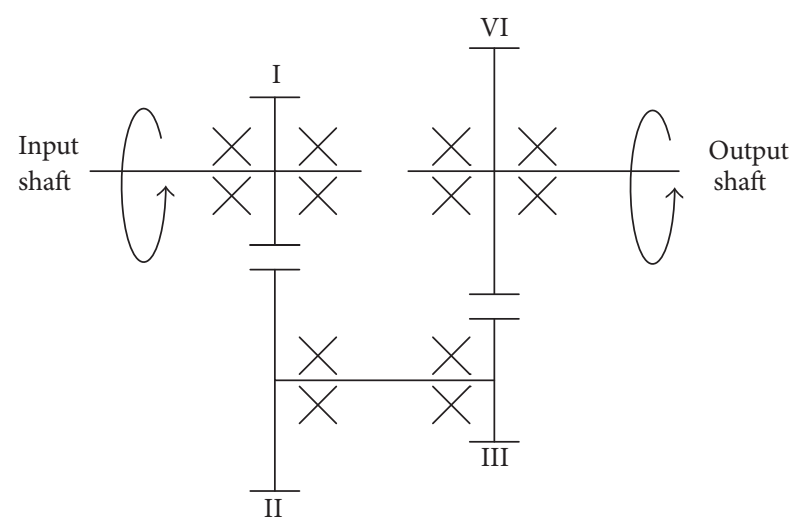

FIGURE 4: Schematic of a two-stage gearbox.

components unrelated to the gear mesh vibration signature can persist also after the TSA. In addition, the proposed signal processing procedure focuses on the local modulation 
of amplitude and phase; hence a low-pass filter is desirable in order to cut off the high frequency signal components that should belong to the impulsive events. Therefore, the signal is conditioned with a low-pass filter with a cut-off order (the signal belongs to the angle domain) equal to 3 times the gear mesh order of the greater gear. Bearing in mind that the filtering process could reduce also some significant components, it is anyway reasonable to assume that the gear mesh vibration signature is properly described taking into account the first 3 meshing gear harmonics (and their sidebands).

The second step consists in the decomposition of the signal and the estimation of the regular signals of gears II and III. EMD, EEMD, and CEEMDAN have been considered, in order to investigate the effects on the use of different EMD algorithms for the signal decomposition. In this work, 500 trials and a fixed white noise standard deviation of 0.2 have been adopted, as suggested in [30, 32]. Furthermore, it has to be remarked that the procedure has been developed in MATLAB environment exploiting the EMD algorithms available at http://perso.ens-lyon.fr/patrick .flandrin/emd.html and http://bioingenieria.edu.ar/grupos/ ldnlys/metorres/re_inter.htm. Instead, the regular signal is defined as the sum of the gear mesh harmonics from the TSA signal. In this application the fundamental gear mesh order and the first two harmonics are taken into account. The meaning of the estimation of the regular signals will be clarified hereafter.

The third step is the core of the proposed methodology. A major issue on the use of the Empirical Mode Decomposition is the physical interpretation of the IMFs. In fact, there are no established procedures for the identification of the meaningful modes for gear applications. Otherwise, the aim of this work is to generate, from the calculated IMF set, two signals representing the gear mesh vibration signals of gear II and gear III, distinctly. Therefore, it is important not just to identify the physically meaningful modes but also to determine if the mode describes the gear mesh vibration signature of gear II, gear III, or neither of these. The selection criterion of the physically meaningful modes developed in this work is based on the Pearson's Correlation Coefficient (PCC) between the regular signals and the IMFs. The PCC is an indicator of the linear correlation between two variables (signals) and conceptually is similar to the normalized crosscorrelation between two signals with zero lag [35]. PCC value $c$ evaluated for discrete dataset $x$ and $y$ of $n$ samples (e.g., the discrete signals in the angle domain) is defined as follows:

$$
c=\frac{\sum_{i=1}^{n}\left(x_{i}-\bar{x}\right)\left(y_{i}-\bar{y}\right)}{\sqrt{\sum_{i=1}^{n}\left(x_{i}-\bar{x}\right)^{2} \sum_{i=1}^{n}\left(y_{i}-\bar{y}\right)^{2}}},
$$

where $\bar{x}$ and $\bar{y}$ are the mean value of $x$ and $y$, respectively. The PCC can take values between -1 and 1 , where positive values mean a direct correlation while negative ones mean inverse correlation. For our purpose, PCC $\geq 0.7$ means a strong linear correlation, $0.3 \leq \mathrm{PCC}<0.7$ means moderate correlation, $0.1 \leq \mathrm{PCC}<0.3$ means weak correlation, and PCC = 0 means no correlation. In this work, the PCC has been exploited as the merit index for the mode selection in order to allocate each IMF to gear II, gear III, or neither of these. Referring to Figure 5, the assignment process regards the mode selection procedure for building the representative signals of gear II and gear III, namely, $M^{\mathrm{II}}(\theta)$ and $M^{\mathrm{III}}(\theta)$, respectively. The selection criterion that evaluates the physical significance of the modes is based on these properties:

(1) If PCC $\geq 0.3$ (which means at least moderate correlation), the mode is assigned to the representative signal set.

(2) If no IMF satisfies the previous properties, the mode having the maximum value of PCC is representative of the gear.

Therefore, referring to Figure 5, the representative vibration signal of gear II, $M^{\mathrm{II}}(\theta)$, is composed of all the modes (called $m_{j}^{\mathrm{II}}(\theta)$ ) satisfying one of these properties and the same occurs for $M^{\mathrm{III}}(\theta)$. The PCCs are evaluated by the regular signal (e.g., an ideal healthy mesh gear vibration) and the IMFs. Since the modes are estimated from the faulty gear vibration signal, a moderate correlation with the regular signal is expected. Thus, property 1 aims to include all the modes showing a moderate correlation with the regular signal having, however, a significant relationship with the gear vibration signature from the physical standpoint. The second property is introduced in order to include at least one IMF also if property 1 is not met.

Lastly, in the fourth step the estimation of $M^{\mathrm{II}}(\theta)$ and $M^{\mathrm{III}}(\theta)$ of gear II and gear III, respectively, is carried out by means of the sum of the selected modes $m_{j}^{\mathrm{II}}(\theta)$ and $m_{j}^{\mathrm{III}}(\theta)$ evaluated in the third step. After a visual inspection of the representative signals, the objective comparison between $M^{\mathrm{II}}(\theta)$ and $M^{\mathrm{III}}(\theta)$ is achieved by means of different CIs. For this purpose, in order to identify localized gear faults, several CIs can be used for the evaluation of the vibration signal peakiness, which is correlated with the severity of the localized fault. In this study, the following standard CIs have been considered: kurtosis and Crest Factor (CF) $[9,36]$. Furthermore, the effectiveness of the proposed CIs, that is, CPF and NSVP, has been tested for the simulated vibration signals and the real case studies.

\section{Application to Simulated Vibration Signals}

In this section, the first subsection regards the mathematical formulation of the meshing vibration signal model with localized fault in the angle domain; in the second subsection, the effectiveness of the proposed methodology will be verified by means of the simulated signals.

4.1. Signal Model Formulation. Several works [34, 37, 38] regarding the time domain vibration signal modeling of gear faults can be found in the literature. Since the proposed method departs from an averaged angle domain vibration signal (TSA), an angle domain model of the meshing vibration signal of a spur gear with $z$ teeth is proposed hereafter. In healthy gearboxes, meshing gear vibration $x$ with respect to angle $\theta$ is mainly composed of harmonics with fundamental 


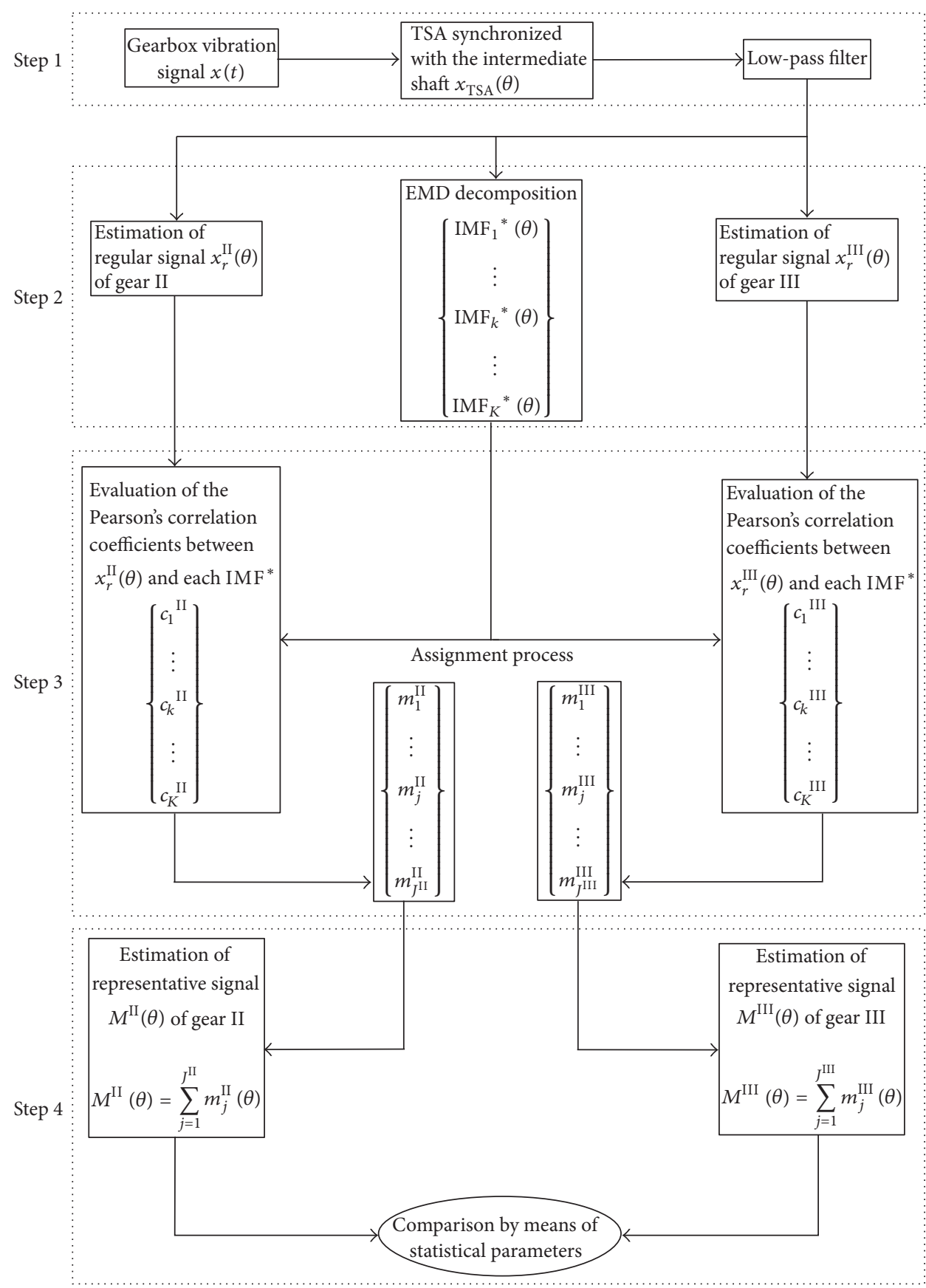

FIGURE 5: Flow-chart of the proposed methodology.

order corresponding to the number of teeth of the gear of interest. For a complete revolution, $x(\theta)$ can be expressed as follows:

$$
x(\theta)=\sum_{n=1}^{N} X_{n} \cos \left(n z \theta+\phi_{n}\right),
$$

where $N$ is the number of harmonics, $X_{n}$ is the amplitude of the $n$th harmonics, $\phi_{n}$ is the phase of the $n$th harmonics, and $z$ is the number of teeth of the gear.
Let us consider the two-stage healthy gearbox shown in Figure 4. In a complete revolution, the angle domain meshing vibration $x(\theta)$ related to the intermediate shaft may be expressed as follows:

$$
\begin{aligned}
x(\theta)= & \sum_{n=1}^{N} X_{\mathrm{II}, n} \cos \left(n z_{\mathrm{II}} \theta+\phi_{\mathrm{II}, n}\right) \\
& +\sum_{n=1}^{N} X_{\mathrm{III}, n} \cos \left(n z_{\mathrm{III}} \theta+\phi_{\mathrm{III}, n}\right),
\end{aligned}
$$


where $N$ is the number of harmonics, $X_{\mathrm{II}, n}$ is the amplitude of the $n$th harmonics of gear II, $\phi_{\mathrm{II}, n}$ is the phase of the $n$th harmonics of gear II, $z_{\text {II }}$ is the number of teeth of gear II, $X_{\text {III }, n}$ is the amplitude of the $n$th harmonics of gear III, $\phi_{\mathrm{III}, n}$ is the phase of the $n$th harmonics of gear III, and $z_{\text {III }}$ is the number of teeth of gear III. Equation (11) states that the meshing vibration signal $x(\theta)$ is composed of the meshing vibration related to gear II and the gear mesh vibration related to gear III since both gears II and III rotate synchronously.

The presence of a localized gear fault causes a change in the vibration signal model that involves a local amplitude modulation, a local phase modulation, and an impulsive component. Hence assuming localized faults in gear II, (11) can be rewritten as

$$
\begin{aligned}
x_{\text {sim }, \mathrm{II}}(\theta)=x^{\mathrm{II}}(\theta)+x^{\mathrm{III}}(\theta)+\delta(\theta) \\
=\sum_{n=1}^{N} X_{\mathrm{II}, n}\left[1+a_{\mathrm{II}, n}(\theta)\right] \cos \left[n z_{\mathrm{II}} \theta+\phi_{\mathrm{II}, n}+b_{n}(\theta)\right] \\
\quad+\sum_{n=1}^{N} X_{\mathrm{III}, n} \cos \left(n z_{\mathrm{III}} \theta+\phi_{\mathrm{III}, n}\right)+\delta(\theta),
\end{aligned}
$$

where $a_{\mathrm{II}, n}$ is the local amplitude modulation function due to localized fault in gear II, $b_{\mathrm{II}, n}$ is the local phase modulation function due to localized fault in gear II, and $\delta$ is the impulsive component due to localized fault in gear II. The local amplitude function $a_{\mathrm{II}, n}$ and phase modulation function $b_{\mathrm{II}, n}$ used in (13) are described as a Gaussian shape window centred at angle $\theta_{0}$ as follows:

$$
\begin{aligned}
& a_{n}(\theta)=A_{n} e^{-\left(\theta-\theta_{0}\right) / \sigma_{a}^{2}} \\
& b_{n}(\theta)=B_{n} e^{-\left(\theta-\theta_{0}\right) / \sigma_{b}^{2}},
\end{aligned}
$$

where $A_{n}$ and $B_{n}$ are the amplitude of the local modulation and $\sigma$ is the window width. In addition, $\delta(\theta)$ (see (13)) is the damped impulse response that takes into account the impulsive component due to the engagement of the faulted tooth. This component is defined as a train of Dirac impulses having unitary amplitude convoluted by an arbitrary impulse response function (in the form of a 3rd FIR filter):

$$
\delta(\theta)=s(\theta) * h,
$$

where $s$ is the train of unitary impulses corresponding to the fault occurrence and $h$ is the impulse response function. Note that (15) is valid only in steady-state conditions. In fact, this formulation keeps its physical meaning only when the

\begin{tabular}{|c|c|c|c|c|c|}
\hline & \multicolumn{2}{|r|}{$x_{\text {sim,II }}(\theta)$} & \multicolumn{3}{|c|}{$x_{\text {sim,III }}(\theta)$} \\
\hline$z$ & & 93 & & 12 & \\
\hline$N$ & & 3 & & 3 & \\
\hline$X$ & {$[6.000$} & 3.639 2.207 & {$[6.000$} & 3.639 & $2.207]$ \\
\hline$A$ & {$[1.075$} & $3.668-4.518$ & {$[1.075$} & 3.668 & -4.518 \\
\hline$B$ & {$[0.862$} & $\begin{array}{ll}0.319 & -1.308\end{array}$ & {$[0.862$} & 0.319 & $-1.308]$ \\
\hline$\sigma_{a}$ & & 0.011 & & 0.087 & \\
\hline$\sigma_{b}$ & & 0.011 & & 0.087 & \\
\hline$\theta_{0}$ & & 174.179 & & 180.000 & \\
\hline$h$ & {$[1.000$} & $-0.300 \quad 0.910$ & {$[1.000$} & -0.300 & 0.910 \\
\hline
\end{tabular}
frequency domain is equivalent to the order domain up to a proportional constant (the fundamental rotation frequency). Hence, this is true only if the rotation frequency of the system is, to a good approximation, constant.
TABLE 1: Simulated signal parameters.

Analogously, the gear mesh vibration model can be written in the case of a localized fault in gear III:

$$
\begin{gathered}
x_{\text {sim }, \mathrm{III}}(\theta)=x^{\mathrm{II}}(\theta)+x^{\mathrm{III}}(\theta)+d(\theta)+\delta(\theta) \\
=\sum_{n=1}^{N} X_{\mathrm{II}, n} \cos \left(n z_{\mathrm{II}} \theta+\phi_{\mathrm{II}, n}\right) \\
+\sum_{n=1}^{N} X_{\mathrm{III}, n}\left[1+a_{\mathrm{III}, n}(\theta)\right] \\
\cdot \cos \left[n z_{\mathrm{III}} \theta+\phi_{\mathrm{III}, n}+b_{n}(\theta)\right]+\delta(\theta) .
\end{gathered}
$$

Referring to the gearbox depicted in Figure 4, (13) and (16) represent the gear mesh vibration models used for the preliminary verification of the methodology.

4.2. Results and Discussion. Two different simulated signals called $x_{\text {sim,II }}(\theta)$ and $x_{\text {sim,III }}(\theta)$ have been considered: the first simulated signal is described in (12) and refers to the case of a localized defect in gear II; the second one is described in (16) and refers to the case of a localized defect in gear III. The parameters used for the MATLAB implementation of $x_{\text {sim,II }}(\theta)$ and $x_{\text {sim,III }}(\theta)$ are reported in detail in Table 1 , while Figure 6 shows the simulated signals. In the first case the localized fault has been simulated on the 45th tooth of gear II whereas in the second case the localized fault has been simulated on the 6th tooth of gear III, which correspond to an angle rotation of about $174 \mathrm{deg}$ and $180 \mathrm{deg}$, respectively.

As mentioned in Section 4.1, the overall simulated signal is a superposition of the vibration signature in the angle domain of the two gears, where the healthy gear is represented by pure tones and the faulty gear is composed of pure tones having local amplitude and phase modulation with the contribution of an impulsive component. De facto, such signals represent a synthesized version of the TSA computed with respect to the intermediate shaft and, according to the properties of the TSA, the contribution of the background noise has been neglected. Considering the proposed diagnostic protocol, Step 1 can be skipped with this vibration signal model since the starting signal is already the TSA of the signal.

The method has been performed using EMD, EEMD, and CEEMDAN in order to investigate the effect of different EMD 


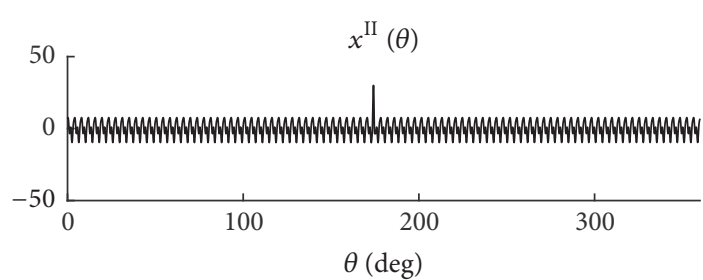

(a)

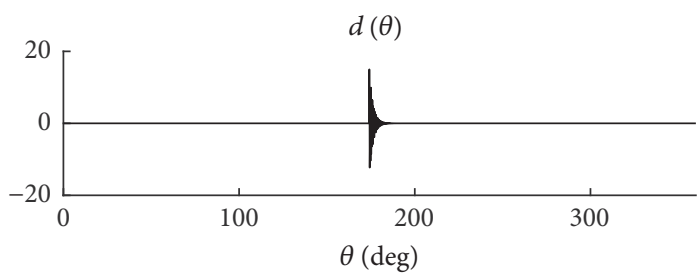

(c)

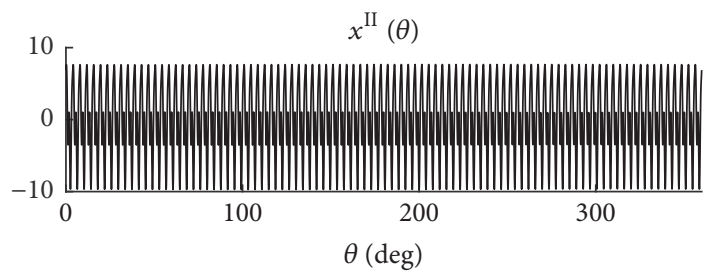

(e)

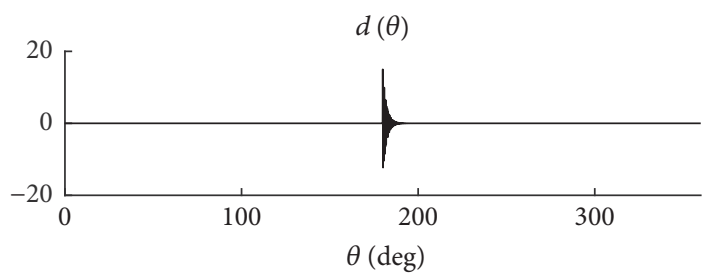

(g)

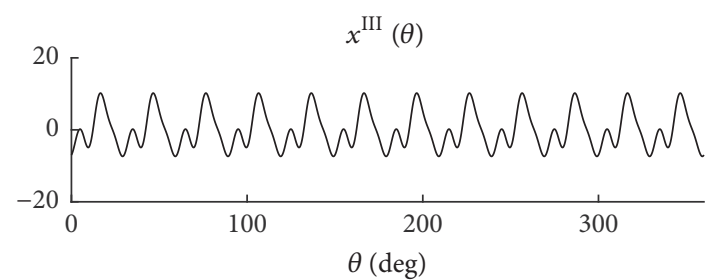

(b)

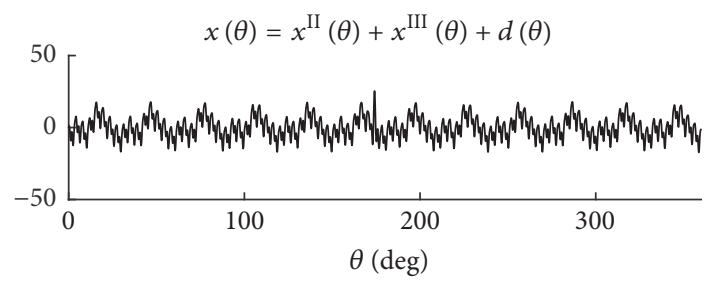

(d)

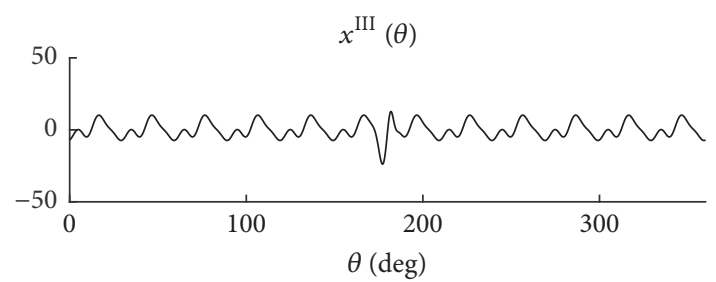

(f)

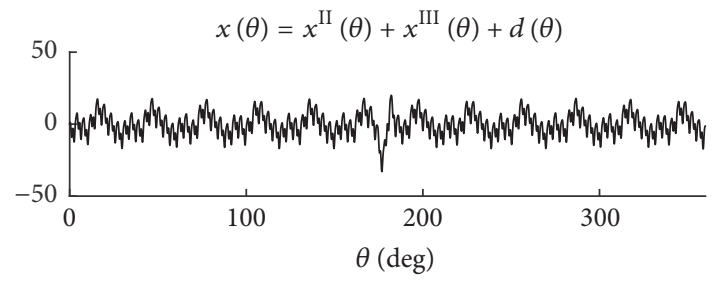

(h)

Figure 6: Simulated signals in the angle domain: (d) $x_{\text {sim,II }}$ and (a-c) its components and (h) $x_{\text {sim,III }}$ and (e-g) its components.

algorithms on the effectiveness of the signal decomposition. In agreement with the settings suggested in the literature, 500 averages and white noise standard deviation of 0.02 have been used for the EEMD and CEEMDAN. The results of the signal decomposition of $x_{\text {sim,II }}(\theta)$ and $x_{\text {sim,III }}(\theta)$ are reported in Figures 7 and 8, respectively. The residue signal, which is a monotonic function, is not displayed since it is not useful for the goal of this work. It should be noted that EMD returns a limited number of modes (5 excluding the residue, i.e., the monotonic mode) whereas the total number of tones present on the simulated signal is 6 since it is equal to the total number of gear mesh harmonics accounted. This behavior can be interpreted as poor quality of the signal decomposition using EMD with respect to EEMD and CEEMDAN.

For the sake of completeness, the estimated PCC values are collected in Figure 9 where the square symbol refers to $c_{i}^{\mathrm{II}}$ and the star symbol refers to $c_{i}^{\mathrm{III}}$. In each diagram, the gray horizontal line refers to the threshold corresponding to PCC $=3$. According to the proposed criterion, the representative signals of gear II and gear III are showed in Figures 10 and 11.
The visual inspection of the representative signals shown in Figure 10 highlights that all the considered EMD algorithms have led to satisfying results. In this case, as expected, representative signal $M^{\mathrm{II}}$ related to the 93 -teeth gear exhibits a localized signal distortion at about $170 \mathrm{deg}$ due to the simulated defect (in agreement to the input data in Table 1) whereas the waveform $M^{\mathrm{III}}$ does not show irregularities. It should be remarked that $M^{\mathrm{III}}$ estimated by the EMD (Figure 10(d)) is less regular than the others just in correspondence to the angle where $M^{\mathrm{II}}$ has the local amplitude/phase modulation. Table 2 collects the statistical indicators estimated by $M^{\mathrm{II}}$ and $M^{\mathrm{III}}$. All the indicators return a positive deviation between the faulty gear and the healthy one, with the only exception of the kurtosis when the EMD is performed; this behavior is in agreement with the observations previously made by the visual inspection of the signal.

Similar remarks can be mentioned by observing Figure 11. Indeed, $M^{\mathrm{III}}$ shows a sudden change of the signal amplitude at about $180 \mathrm{deg}$ that corresponds to the position of the simulated fault (see Table 1). Again, the waveform of $M^{\mathrm{II}}$ does 

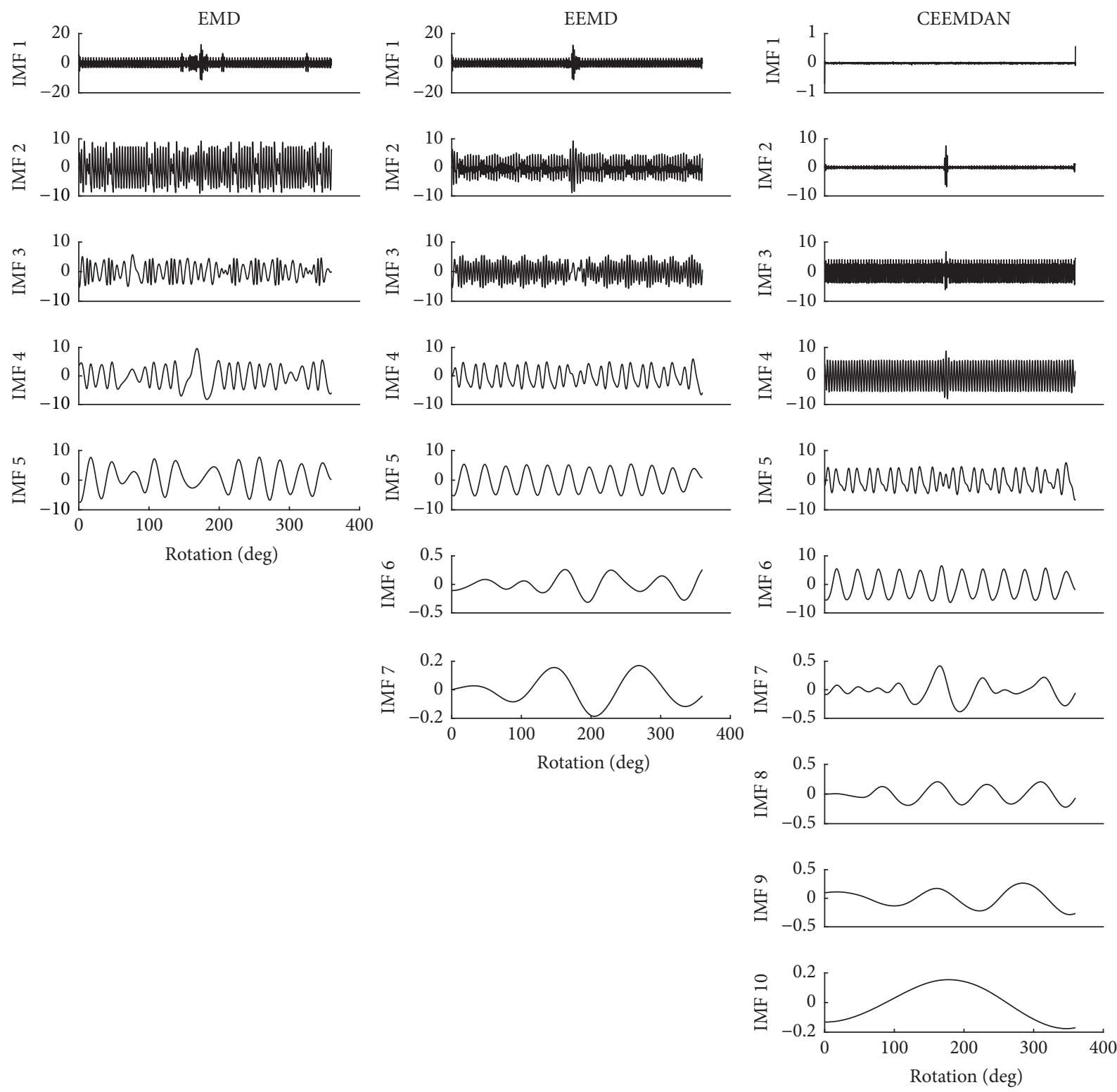

FIgURE 7: IMF sets of $x_{\text {sim,II }}$ obtained by using EMD, EEMD, and CEEMDAN.

not exhibit any abrupt change, although the EMD returns a quiet irregular waveform with respect to the other EMD algorithms. In fact, the indicators collected in Table 3 highlight that negative percentage differences are present only for the EMD, as expected. However, the method performed using the EEMD and the CEEMDAN has allowed clearly identifying the faulty gear both by visual inspection and by comparing condition indicators.

Finally, the validation of the method by simulated signals has pointed out the following aspects:

(i) The signal decomposition by means of the EMD is the worst one among the other EMD algorithms.

(ii) The faulty gear has been correctly identified in both the simulated cases.

(iii) NSVP is the most sensitive indicator.

\section{Application to Real Vibration Signals in the Case of Localized Gear Faults}

In the current section, the proposed methodology has been performed in 2 different cases' studies, discussing the main results. Section 5.1 concerns the investigation of the method using a dedicated gear test bench whereas Section 5.2 regards a more complex transmission mounted on a test rig. The main results have been discussed, focusing on the effectiveness of the proposed methodology performed using actual vibration signals.

5.1. Case 1. The first case study is a two-stage gearbox mounted on a dedicated test rig shown in Figure 12(a) located at the Engineering Department of the University of Ferrara. Detailed information about this test rig can be found in 

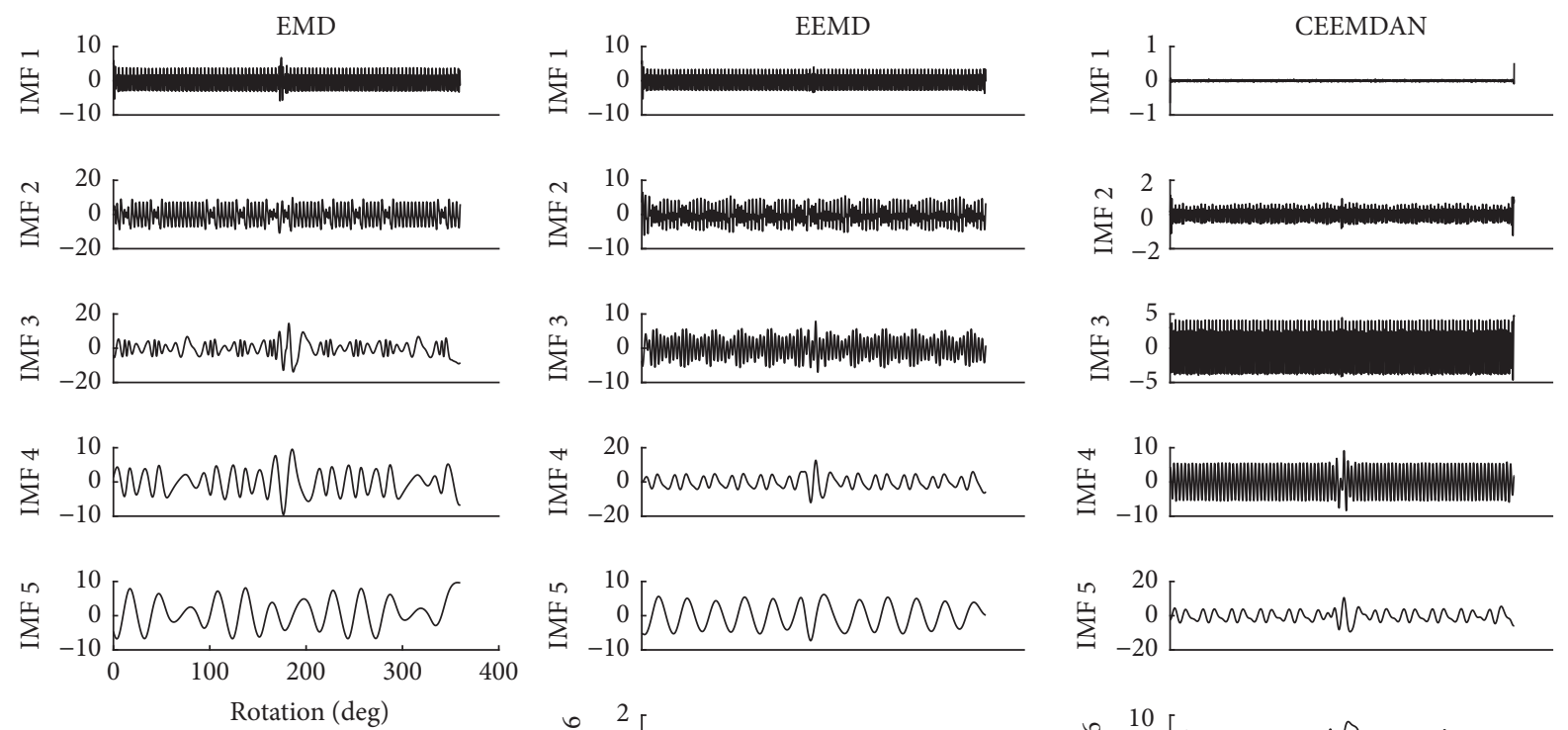

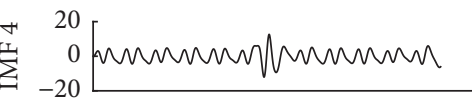
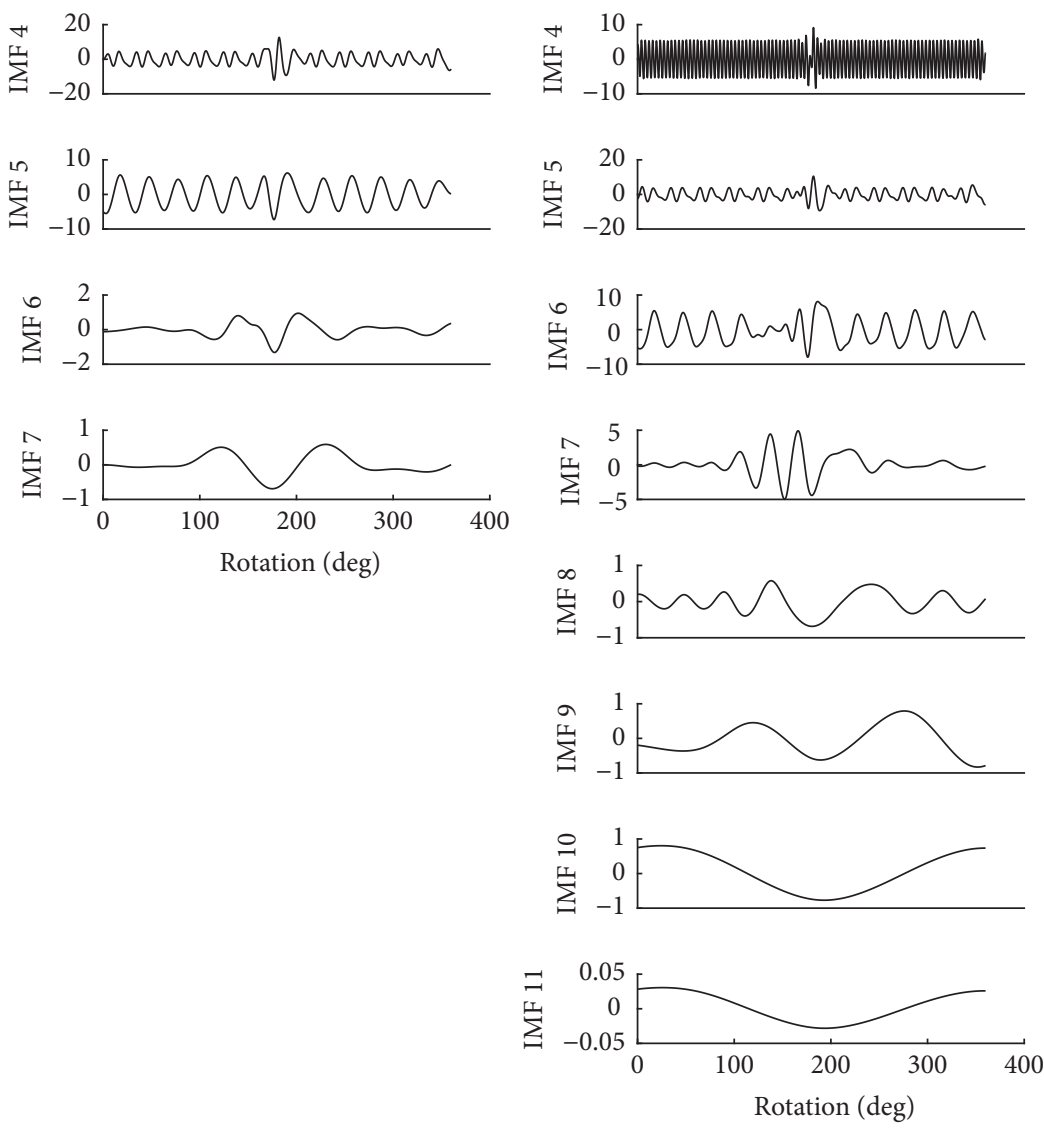

FIGURE 8: IMF sets of $x_{\text {sim,III }}$ obtained by using EMD, EEMD, and CEEMDAN.

TABLE 2: CIs for the simulated signal with localized fault in gear II.

\begin{tabular}{|c|c|c|c|c|c|}
\hline & & Kurtosis & $\mathrm{CF}$ & $\mathrm{CPF}$ & NSVP \\
\hline \multirow{3}{*}{ EMD } & Gear II (faulty) & 2.395 & 4.017 & 1.854 & 0.310 \\
\hline & Gear III (healthy) & 2.742 & 2.673 & 1.083 & 0.0796 \\
\hline & Gear II versus gear III [\%] & -12.671 & 50.269 & 71.244 & 291.452 \\
\hline \multirow{3}{*}{ EEMD } & Gear II (faulty) & 2.484 & 4.494 & 1.872 & 0.173 \\
\hline & Gear III (healthy) & 2.165 & 2.014 & 1.036 & 0.022 \\
\hline & Gear II versus gear III [\%] & 14.713 & 123.180 & 80.722 & 667.777 \\
\hline \multirow{3}{*}{ CEEMDAN } & Gear II (faulty) & 2.340 & 4.284 & 1.857 & 0.205 \\
\hline & Gear III (healthy) & 2.177 & 1.992 & 1.027 & 0.016 \\
\hline & Gear II versus gear III [\%] & 7.487 & 115.058 & 80.777 & 1156.891 \\
\hline
\end{tabular}




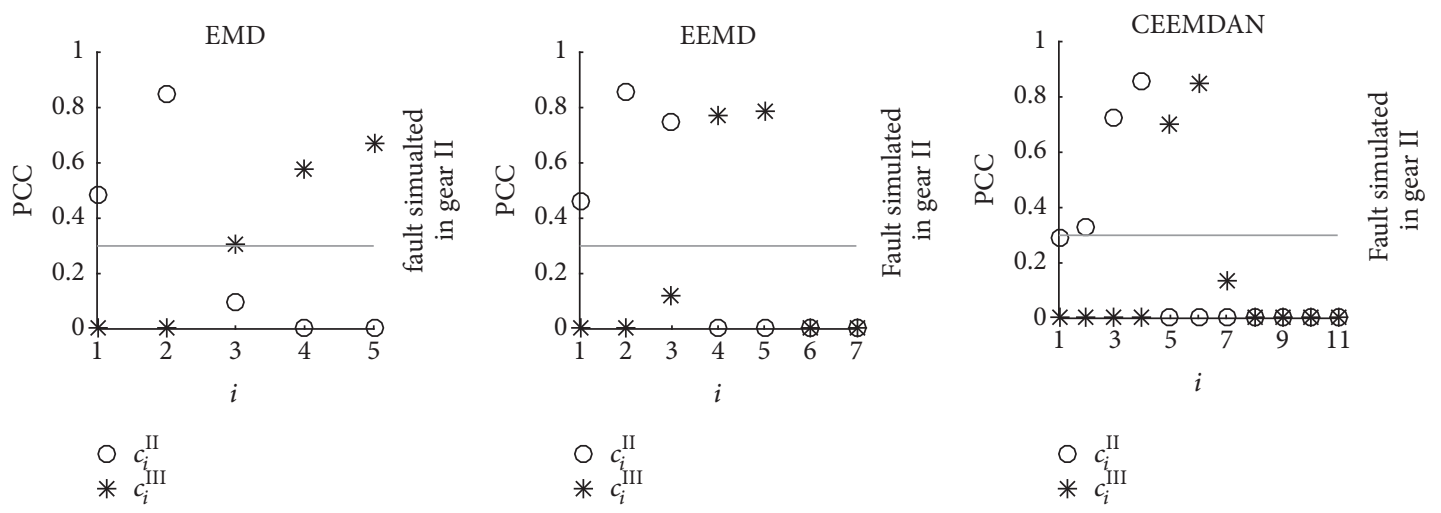

(a)

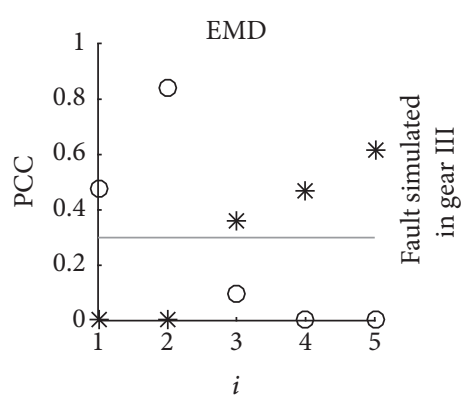

$\begin{array}{ll}O & c_{i}^{\mathrm{II}} \\ * & c_{i}^{\mathrm{III}}\end{array}$ (b)

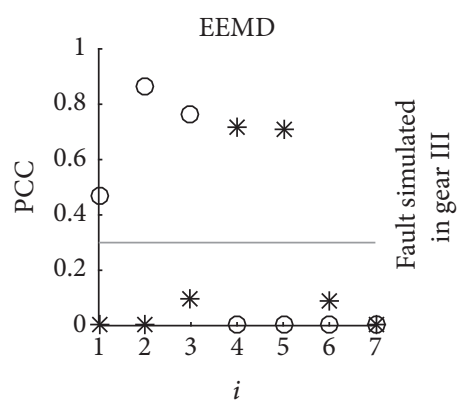

$\begin{array}{ll}\mathrm{O} & c_{i}^{\mathrm{II}} \\ * & c_{i}^{\mathrm{II}}\end{array}$

(e) (c)

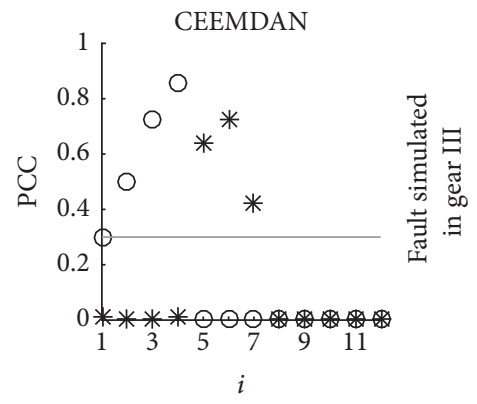

$\begin{array}{ll}\mathrm{O} & c_{i}^{\mathrm{II}} \\ * & c_{i}^{\mathrm{III}}\end{array}$

(f)

FIGURE 9: PCC values $(\mathrm{a}-\mathrm{c})$ in the case of the simulated signal with localized fault in gear II and (d-e) in the case of the simulated signal with localized fault in gear III.

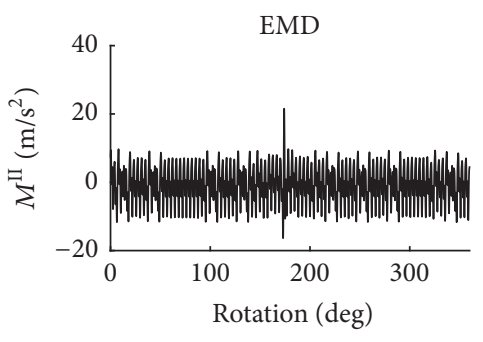

(a)

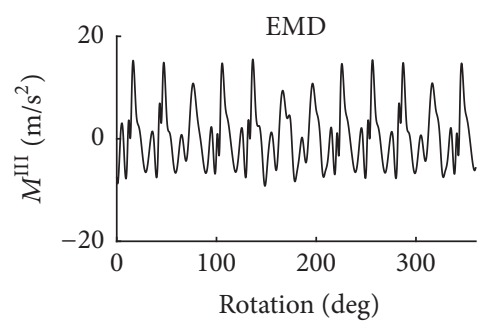

(d)

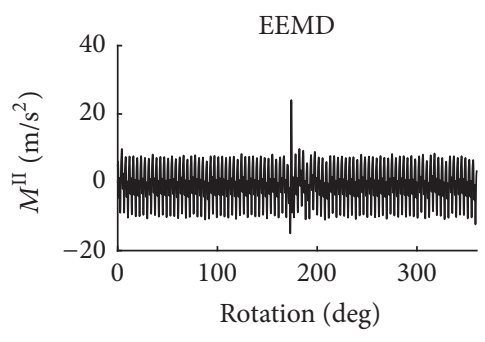

(b)

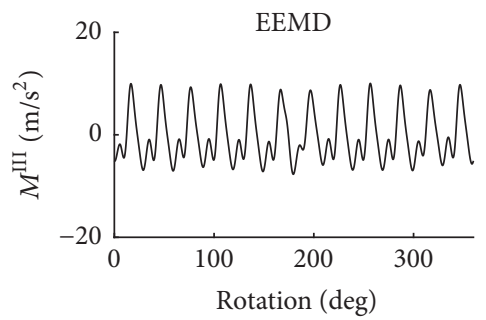

(e)

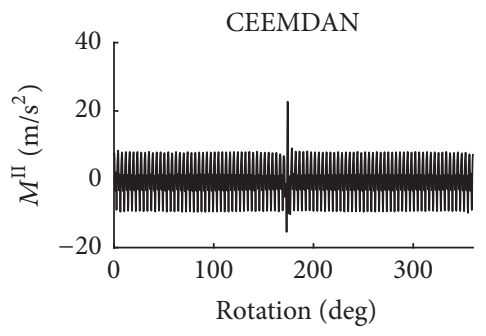

(c)

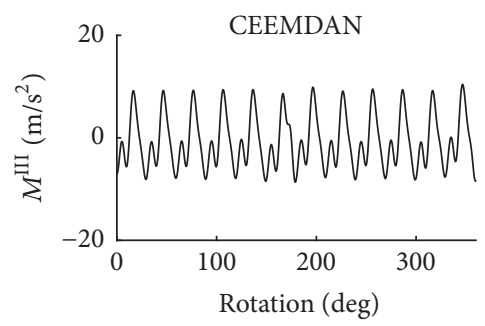

(f)

FIGURE 10: Representative signals for $(\mathrm{a}-\mathrm{c})$ gear II and $(\mathrm{d}-\mathrm{f})$ gear III in the case of the simulated signal with localized fault in gear II. 


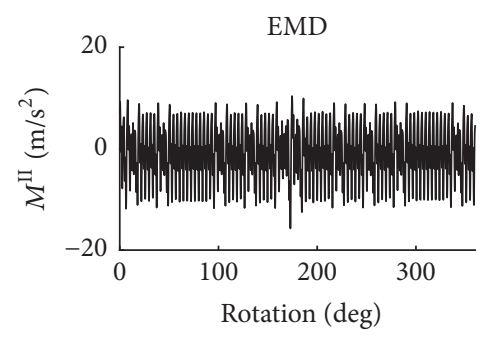

(a)

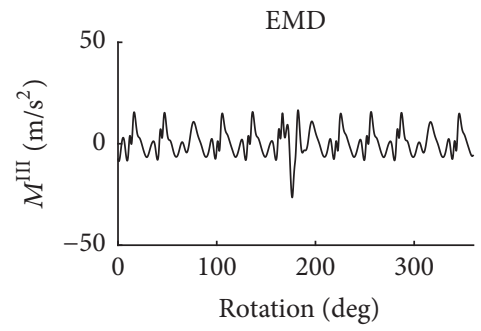

(d)

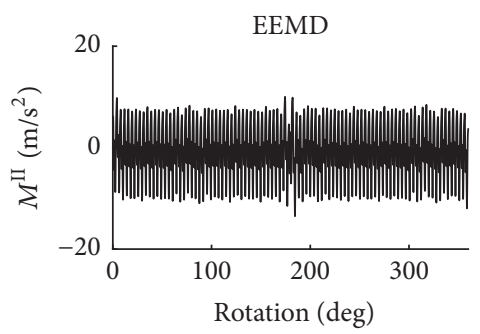

(b)

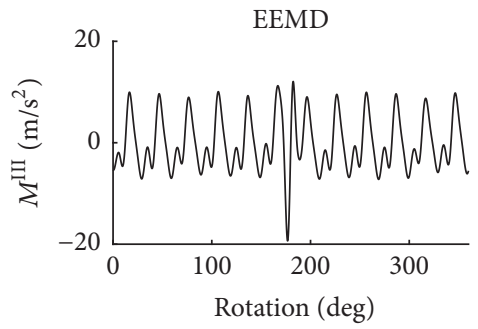

(e)

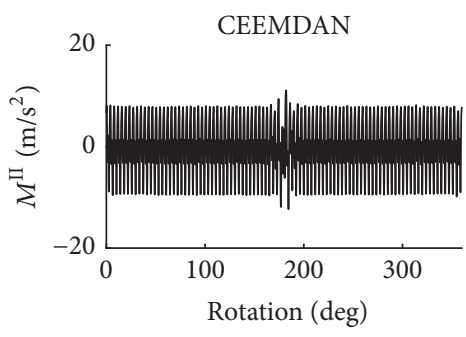

(c)

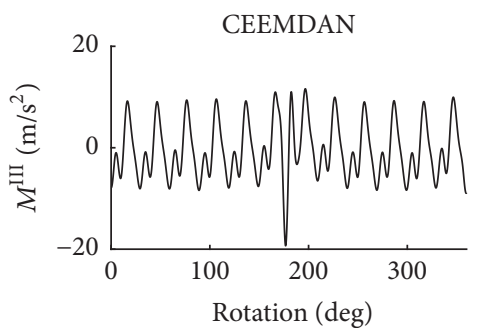

(f)

FIGURE 11: Representative signals for $(\mathrm{a}-\mathrm{c})$ gear II and $(\mathrm{d}-\mathrm{f})$ gear III in the case of the simulated signal with localized fault in gear III.

TABLE 3: CIs for the simulated signal with localized fault in gear III.

\begin{tabular}{|c|c|c|c|c|c|}
\hline & & Kurtosis & $\mathrm{CF}$ & $\mathrm{CPF}$ & NSVP \\
\hline \multirow{3}{*}{ EMD } & Gear II (healthy) & 2.167 & 2.989 & 1.625 & 0.288 \\
\hline & Gear III (faulty) & 3.980 & 4.146 & 1.579 & 0.261 \\
\hline & Gear III versus gear II [\%] & 83.697 & 38.733 & -2.756 & -9.628 \\
\hline \multirow{3}{*}{ EEMD } & Gear II (healthy) & 2.062 & 2.569 & 1.329 & 0.007 \\
\hline & Gear III (faulty) & 2.855 & 3.578 & 1.562 & 0.334 \\
\hline & Gear III versus gear II [\%] & 38.482 & 39.266 & 17.488 & 4958.513 \\
\hline \multirow{3}{*}{ CEEMDAN } & Gear II (healthy) & 2.039 & 2.357 & 1.309 & 0.013 \\
\hline & Gear III (faulty) & 2.647 & 3.428 & 1.469 & 0.351 \\
\hline & Gear III versus gear II [\%] & 29.847 & 45.404 & 12.228 & 2528.605 \\
\hline
\end{tabular}

[39]. The investigated gearbox is composed of two stages of helical gears: the first one having 18 and 71 teeth whereas the second one has 12 and 55 teeth. Hence, referring to the gearbox scheme in Figure 4, gear II and gear III have 71 teeth and 12 teeth, respectively. The localized fault, namely a gear tooth spall, has been artificially seeded on the 71-teeth gear, as shown in Figure 12(b). The test has been carried out in steadystate condition at $3600 \mathrm{rpm}$ using a nominal load of $48.8 \mathrm{Nm}$.

The vibration signals in the radial direction have been collected by means of B\&K piezoelectric accelerometer type 4943 placed on the bearing support of the first stage pinion with sampling frequency $12.4 \mathrm{kHz}$ for a total time length of $4 \mathrm{~s}$ while the input shaft speed has been measured by a tachometer sensor.

Figure 13 collects the TSA of the measured vibration signal as well as its spectrogram. The TSA has been performed in the angular domain taking into account 4260 points per revolution. The localized fault is easy to recognize on both the diagrams since it appears as a sudden increase of the signal amplitude in a slight rotation range. Thus, even if the presence of the gear tooth spall is obvious, these approaches are not able to identify which gear owns the fault.
As done before, three different EMD algorithms have been considered in order to verify the sensitivity of the final results with respect to the adopted EMD method. The signal decomposition has been performed using the same settings reported in Section 4.2 and the results are collected in Figure 14. According to the PCC values collected in Figure 15, the representative signals of gear II and gear III have been computed, as reported in Figure 16. From the visual inspection of the representative signals in Figure 16 it is not hard to identify the faulty gear. Indeed, the waveform related to the 71-teeth wheel exhibits a large amplitude increase at $170 \mathrm{deg}$ that is the effect of the engagement of the faulty tooth. However, Figures 16(d) and 16(e) show a sudden change of the signal amplitude at about $170 \mathrm{deg}$ that corresponds exactly to the angle position of the defect in gear II (clearly visible in Figures 16(a)-16(c) and in the TSA in Figure 13(a)). The diagram related to the CEEMDAN in Figure 16(e) actually displays a local change of amplitude too. However it should be noted that such a change occurs at about $250 \mathrm{deg}$, which is not in agreement with the fault position shown on the TSA of the signal. 


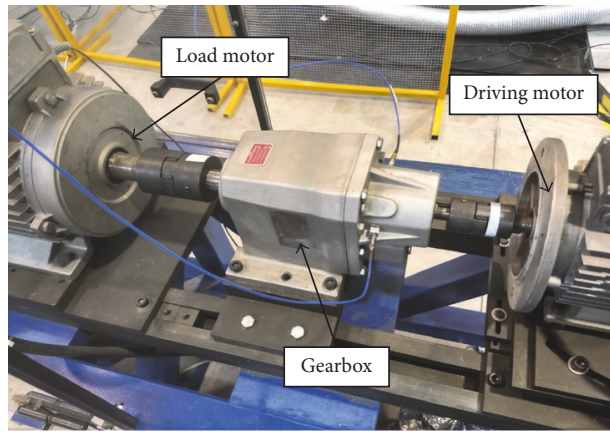

(a)

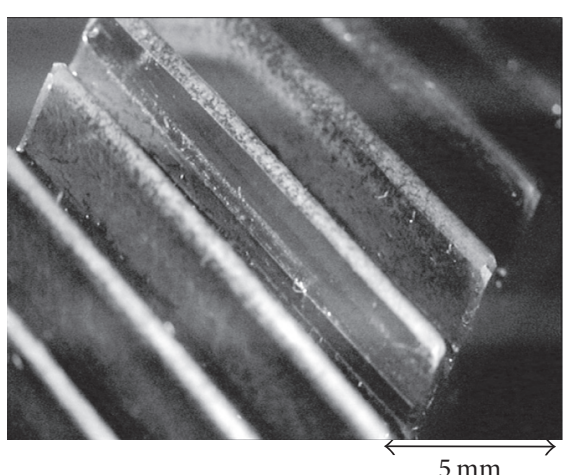

(b)

Figure 12: (a) Experimental setup of Case 1 and (b) gear with a spalled tooth.

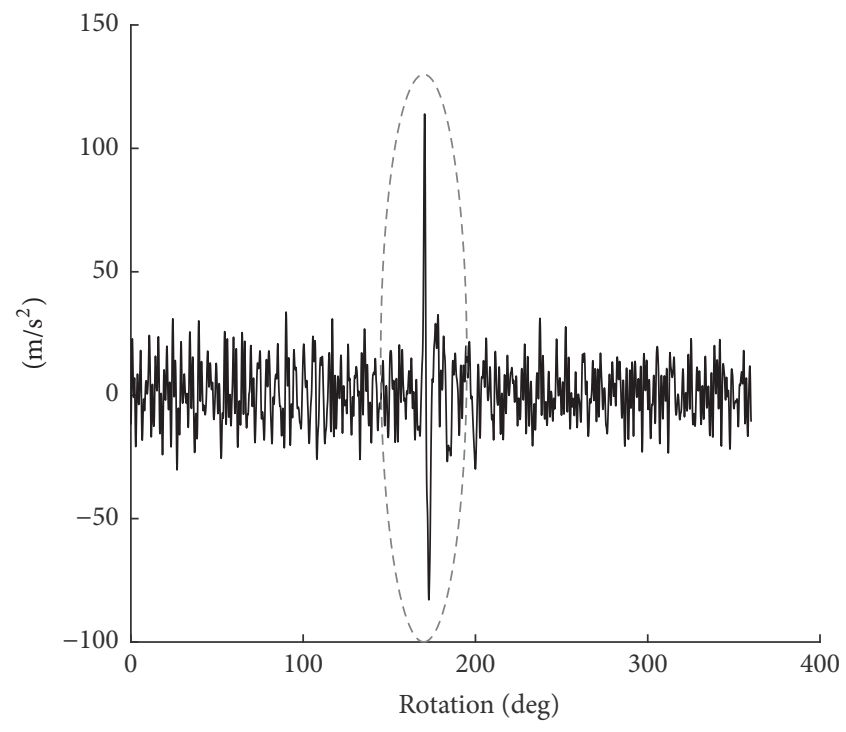

(a)

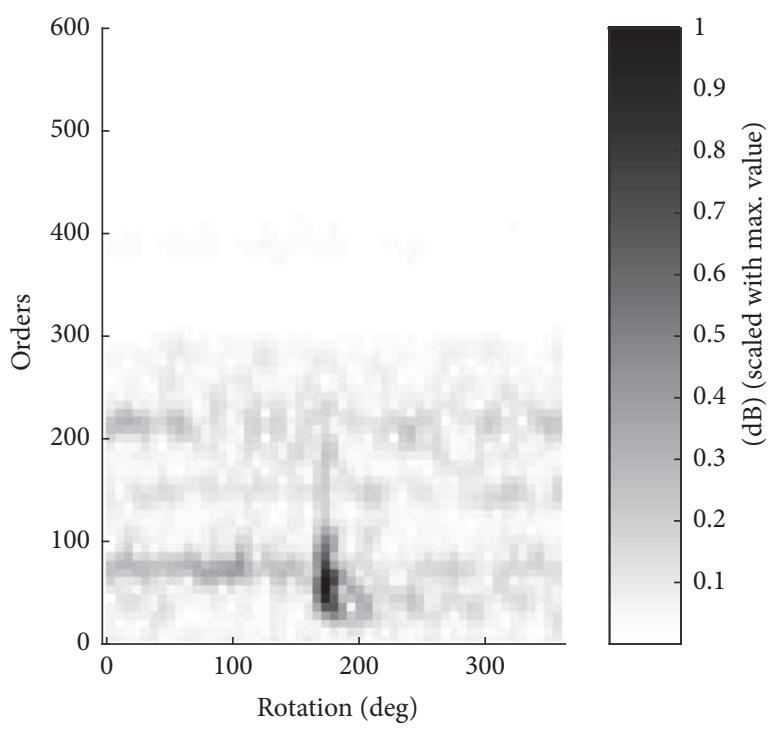

(b)

Figure 13: (a) Time Synchronous Average related to the intermediate shaft of Case 1 (the dashed circle highlights the impulsive signal component due to the damaged tooth engagement) and (b) the related spectrogram (300 samples of window length and $75 \%$ overlap).

Unfortunately, the visual inspection of the signal can be open to different interpretations; thus the fault identification is determined by the comparison of condition indicators. Table 4 highlights a significant difference between gear II and gear III, with a minimum percentage difference of 93.872. The proposed CIs (i.e., CPF and NSVP) are the most sensitive to the presence of an impulsive component in the vibration signature as demonstrated by the larger percentage difference with respect to the other traditional CIs.

Finally, in this first experimental case the proposed method is effective in the identification of the faulty gear. Moreover, CEEMDAN is the EMD algorithm that returns the best result taking into account the CIs values as well as the waveform of the representative signals.

5.2. Case 2. The second case study concerns a more complex gearbox driven by an asynchronous motor. Figure 17 shows the experimental setup: the time domain vibration signal in the radial direction has been acquired by a monoaxial piezoelectric accelerometer (PCB 353B18) with a sampling frequency of $25.6 \mathrm{kHz}$, while the tachometer signal has been simultaneously collected using a tachometer probe with zebra tape. The transmission exhibits abnormal loudness due to a localized gear fault on the two-stage gearbox just after the input cardan shaft (see Figure 17(b)). The steady-state operational test has been carried out at $600 \mathrm{rpm}$ at the input cardan shaft. Considering the gearbox layout in Figure 4, gear II has 92 teeth whereas gear III has 10 teeth.

Gear II presents a bump on a tooth flank caused by the handling during the surface hardening process. Such a faulty tooth flank engages only in the reverse motion and it has been verified by visual inspection. Furthermore, such a natural defect is clearly visible in the TSA signal performed on the intermediate shaft using 3680 samples per revolution, as reported in Figure 18(a). The presence of the fault is clear also on the spectrogram as well in Figure 18(b). As in Case 1, 
EMD$$
\text { 岳 }
$$$$
\sum_{-1}^{-1}
$$
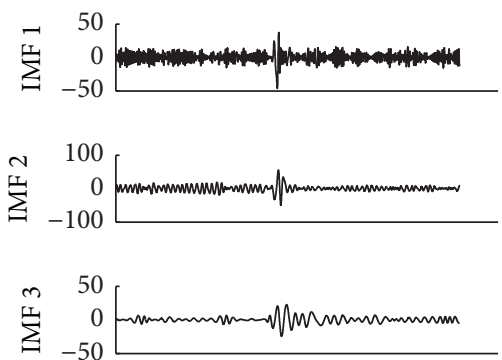

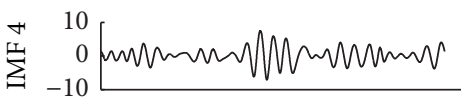

$\sum_{-5}^{n} n_{n}^{5}$
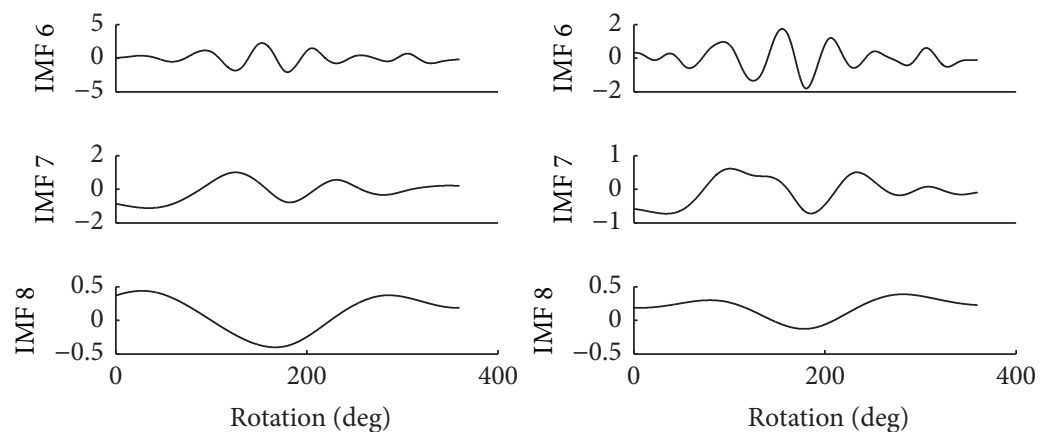

EEMD
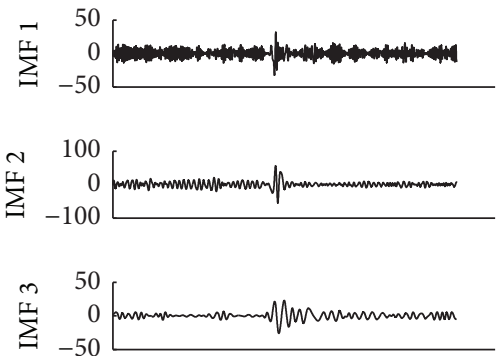

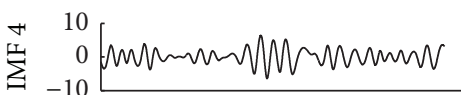

$\sum_{-5}^{n} \begin{aligned} & 5 \\ & 0\end{aligned}$
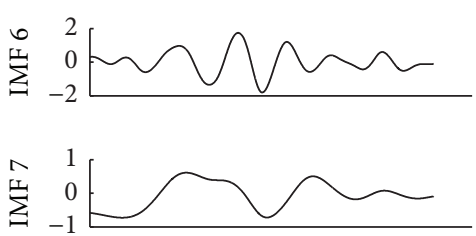

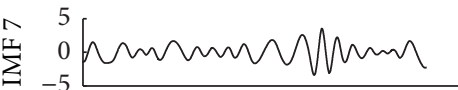
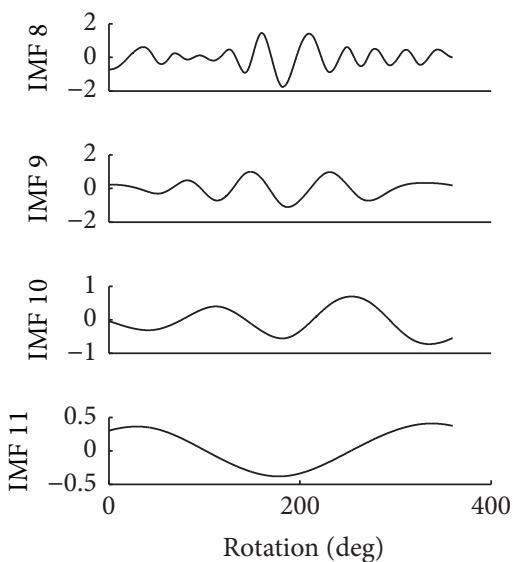

FIGURE 14: IMF sets of Case 1 obtained by using EMD, EEMD, and CEEMDAN.

TABLE 4: CIs for the vibration signal of Case 1, with localized fault in gear II.

\begin{tabular}{|c|c|c|c|c|c|}
\hline & & Kurtosis & $\mathrm{CF}$ & $\mathrm{CPF}$ & NSVP \\
\hline \multirow{3}{*}{ EMD } & Gear II (faulty) & 10.220 & 8.785 & 4.437 & 1.395 \\
\hline & Gear III (healthy) & 3.785 & 8.021 & 2.288 & 0.251 \\
\hline & Gear II versus gear III [\%] & 170.043 & 142.209 & 93.872 & 457.089 \\
\hline \multirow{3}{*}{ EEMD } & Gear II (faulty) & 9.945 & 7.806 & 4.444 & 1.205 \\
\hline & Gear III (healthy) & 2.962 & 2.931 & 1.942 & 0.309 \\
\hline & Gear II versus gear III [\%] & 235.745 & 166.309 & 128.859 & 290.713 \\
\hline \multirow{3}{*}{ CEEMDAN } & Gear II (faulty) & 8.031 & 7.177 & 4.332 & 1.139 \\
\hline & Gear III (healthy) & 3.141 & 3.036 & 2.141 & 0.112 \\
\hline & Gear II versus gear III [\%] & 155.666 & 136.382 & 102.324 & 917.173 \\
\hline
\end{tabular}




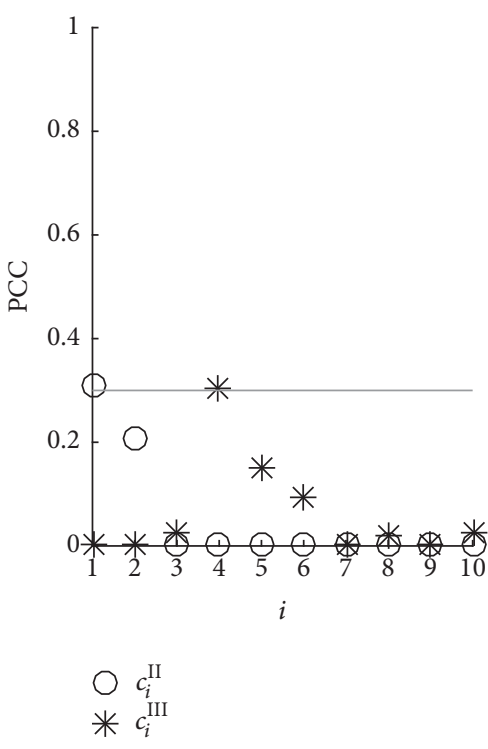

(a)

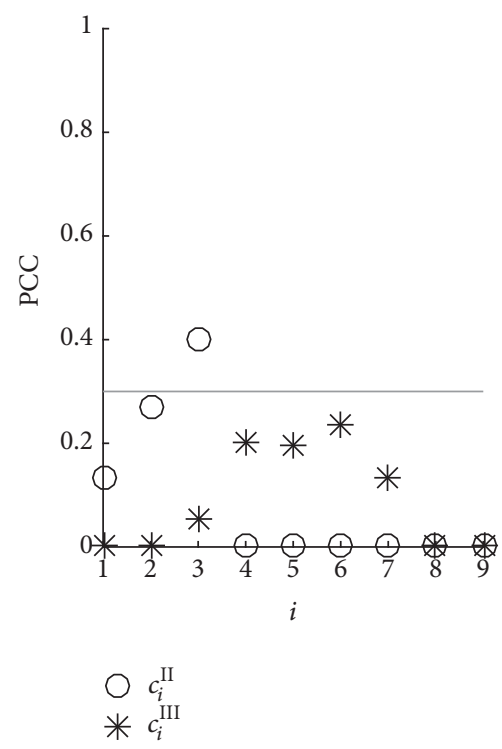

(b)

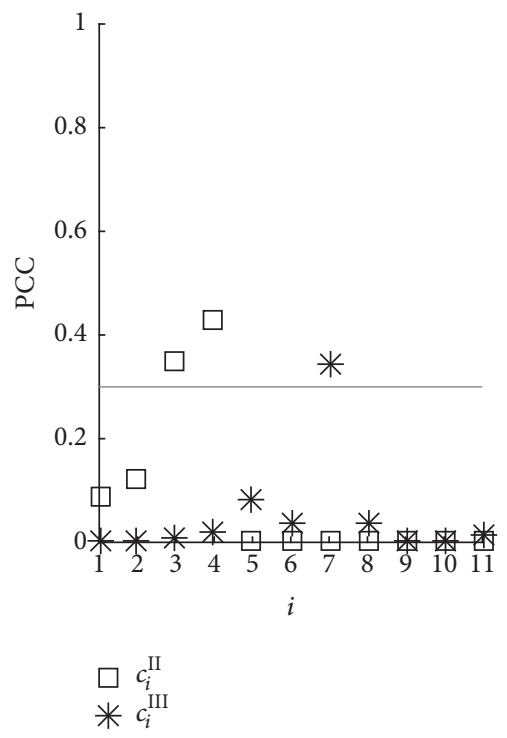

(c)

Figure 15: PCC values of Case 1 estimated taking into account (a) EMD, (b) EEMD, and (c) CEEMDAN.

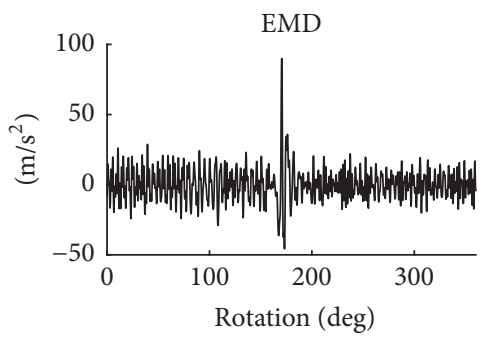

(a)

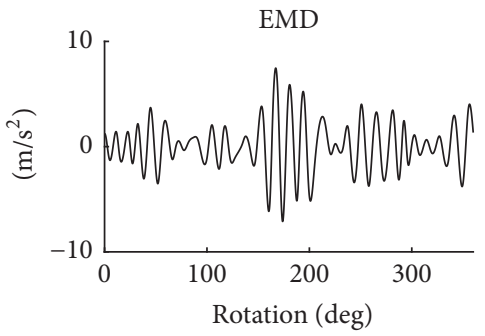

(d)

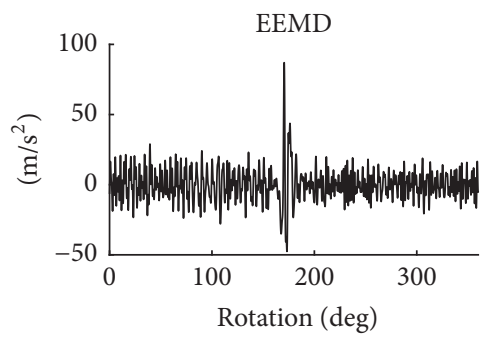

(b)

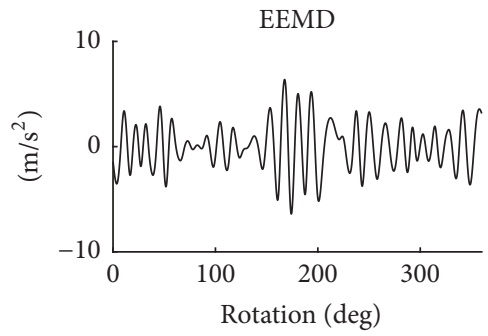

(e)

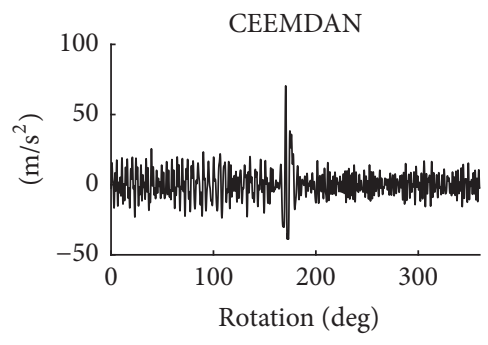

(c)

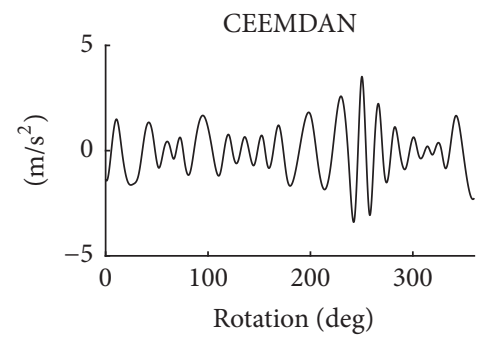

(f)

FIGURE 16: Representative signals of $(\mathrm{a}-\mathrm{c})$ gear II and $(\mathrm{d}-\mathrm{f})$ gear III for Case 1.

these signal processing techniques are not able to identify if the defect is related to the gear of 92 or 10 teeth.

Again, this experimental case study has been investigated by using the same modus operandi of Case 1. For the sake of completeness, signal decomposition results and the PCC values for the estimation of the representative signals are reported in Figures 19 and 20, respectively. By going directly to the visual inspection of the representative signals (Figure 21), it can be noted that in this case the different EMD algorithms have a significant impact on the final results of the methods. From the physical standpoint, the representative signal related to gear II (see Figures 21(a)-21(c)) correctly reflects the presence of the fault at about $170 \mathrm{deg}$ due to the localized increase of the signal amplitude. However, this behavior is also present on the representative signal related to gear III computed with the EMD and the EEMD (see Figures $21(\mathrm{~d})$ and $21(\mathrm{e}))$. On the other hand, the representative signals estimated with the CEEMDAN are easy to interpret since the signal related to gear III (Figure 21(f)) does not contain any remarkable local change of amplitude that can be attributed 


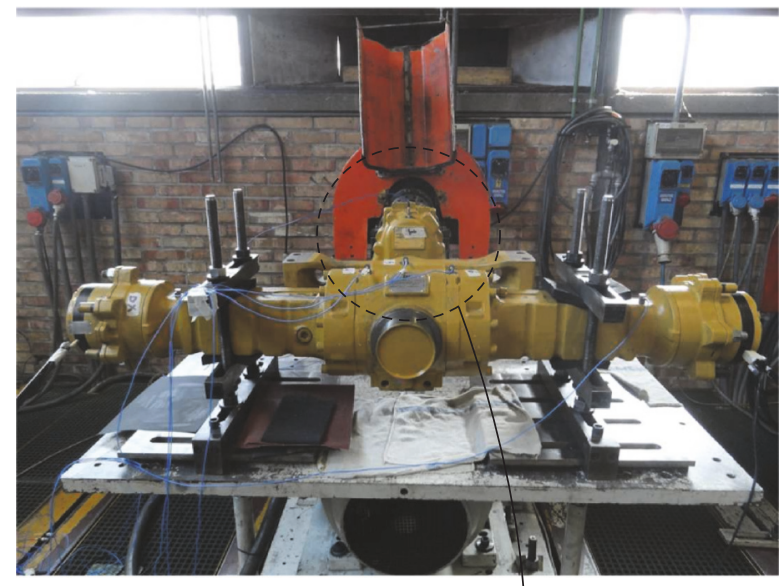

(a)

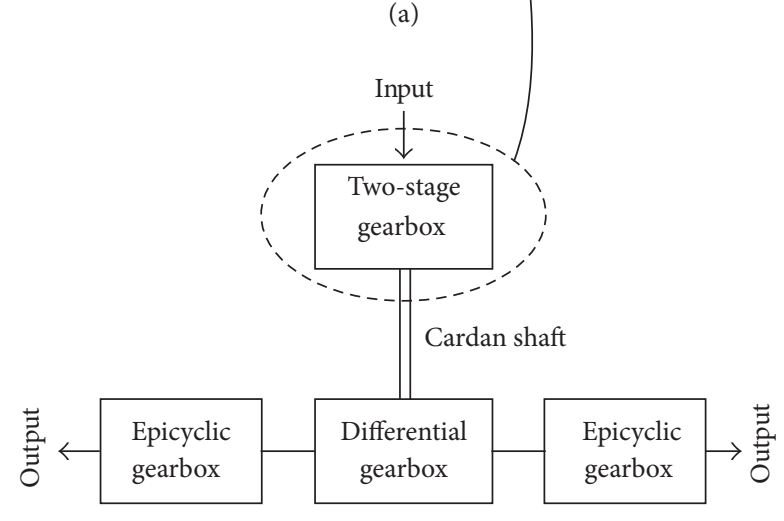

(b)

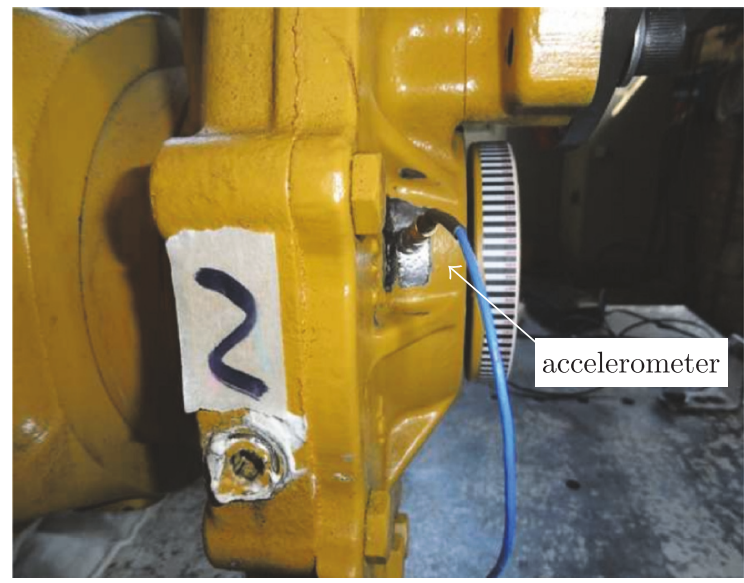

(c)

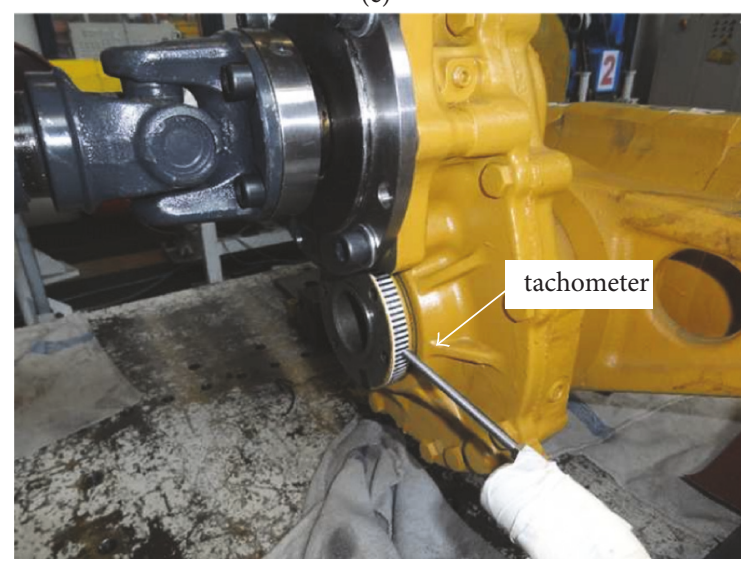

(d)

FIGURE 17: Experimental setup of Case 1: (a) transmission system on the test bench, (b) schematic of the transmission, (c) accelerometer, and (d) tacho probe with zebra tape.

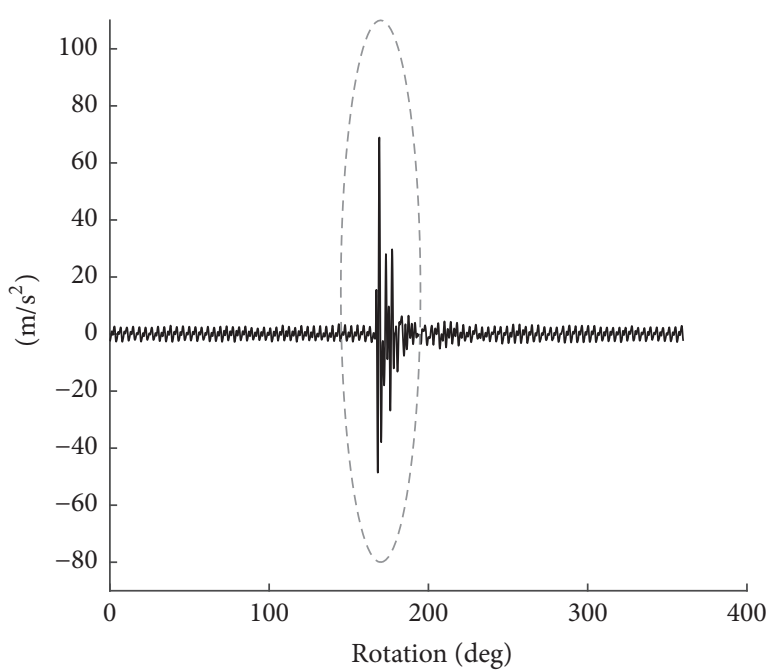

(a)

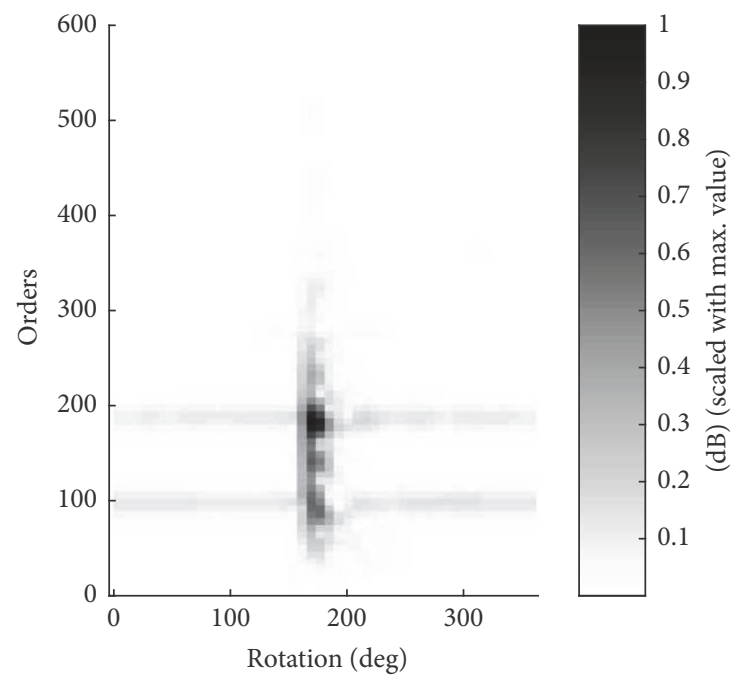

(b)

Figure 18: (a) Time Synchronous Average related to the intermediate shaft of Case 2 (the dashed circle highlights the impulsive signal component due to the damaged tooth engagement) and (b) the related spectrogram (225 samples of window length and $75 \%$ overlap). 

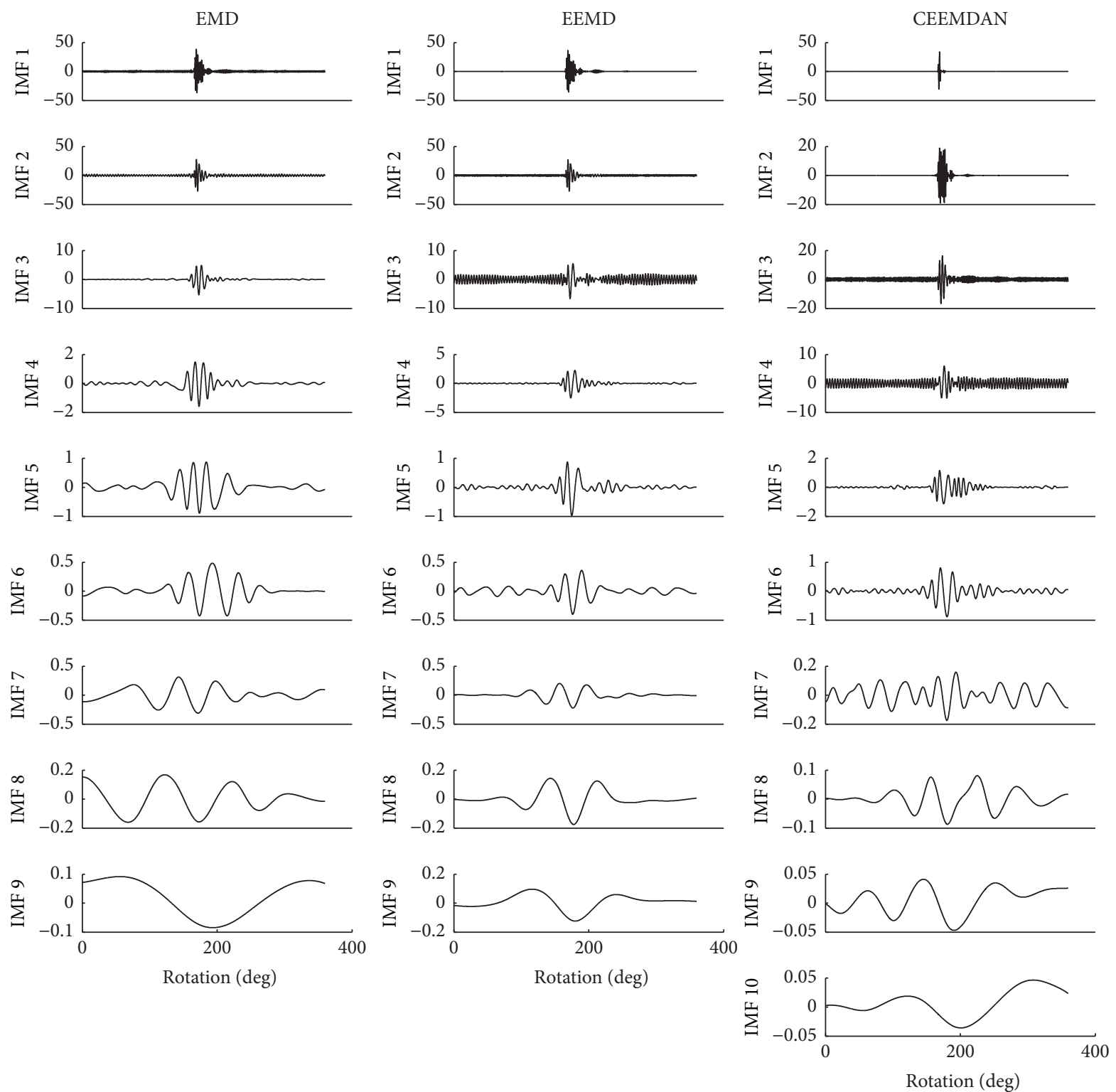

Figure 19: IMF sets of Case 2 obtained by using EMD, EEMD, and CEEMDAN.

to a localized gear fault. Therefore, the only EMD algorithm that allows a clear visual interpretation of the signals is the CEEMDAN.

The remarks gathered by the visual inspection of the signal can be confirmed by analyzing the CIs collected in Table 5. In fact, the higher percentage differences between the faulty gear and the healthy one are achieved considering the CEEMDAN. Furthermore, it should be noted that the results obtained with the EEMD are not satisfying since the percentage difference is low for the CF and CPF while the kurtosis completely fails on the identification of the faulty gear.

Although this experimental case has been more difficult to handle than the first one, the methodology has provided a correct result when the CEEMDAN is performed. The use of several CIs allows defining an objective criterion in order to define which gear is faulty, reducing the error due to the user interpretation.

\section{Conclusions}

Commonly, the gear fault detection is restricted to the identification of the stage containing the faulty gear rather than the faulty gear itself. However, the exact knowledge of the faulty gear is of prime importance in industrial applications. The goal is to propose a methodology being able to overcome the limit of traditional signal processing techniques that detect just the stage of the faulty gear. For this purpose, a EMDbased methodology has been presented for the local gear fault diagnosis, proposing also two new condition indicators based on the RMS values estimated on the angular pitch rather than the entire vibration signal. 


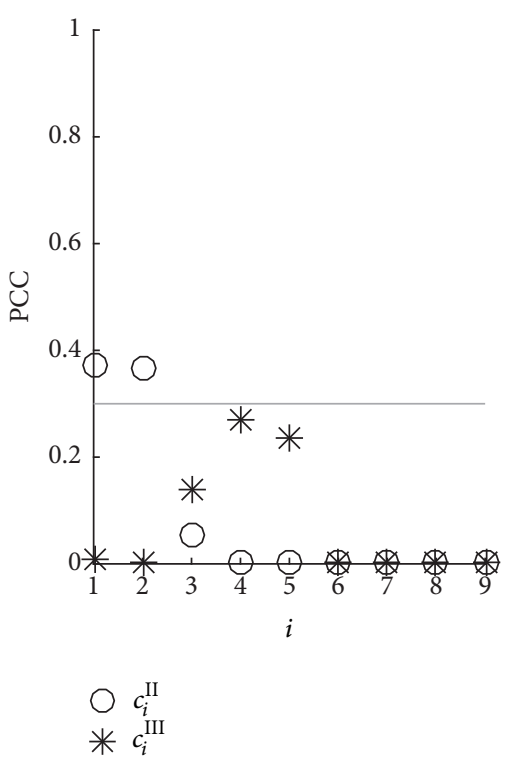

(a)

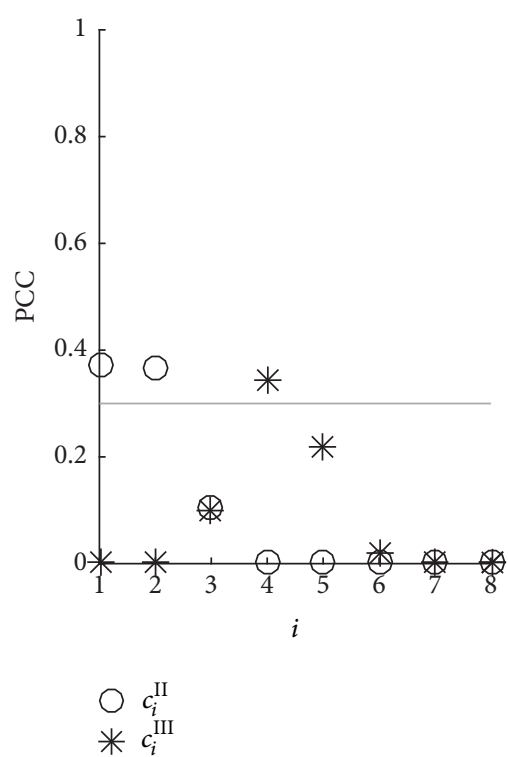

(b)

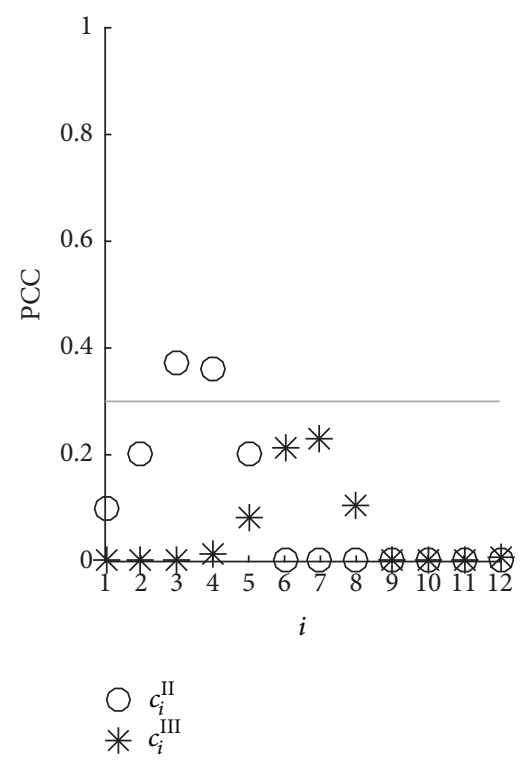

(c)

FIgUre 20: PCC values of Case 2 estimated taking into account (a) EMD, (b) EEMD, and (c) CEEMDAN.

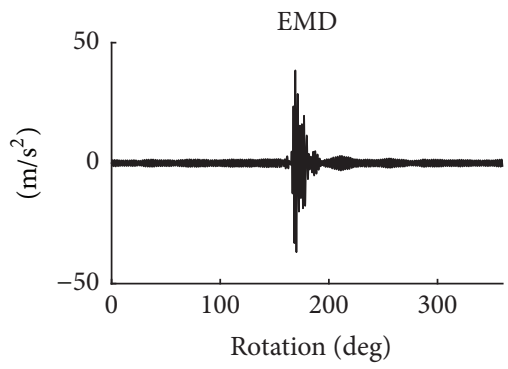

(a)

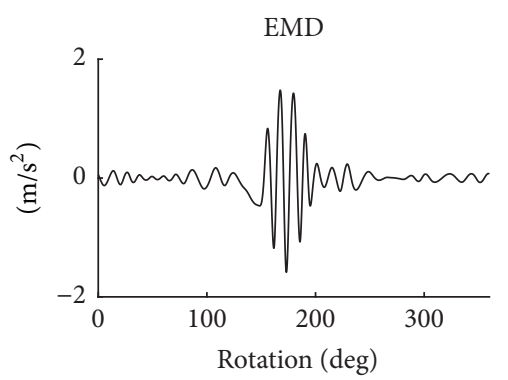

(d)

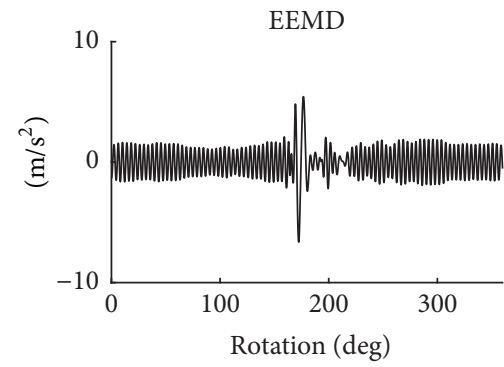

(b)

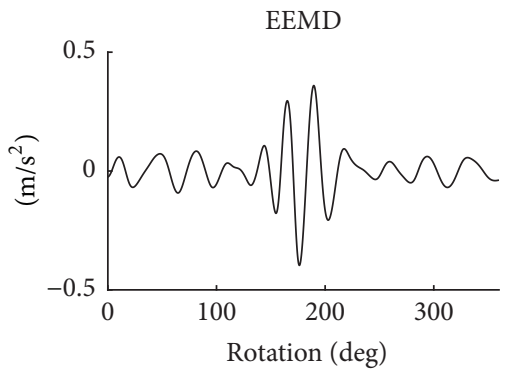

(e)

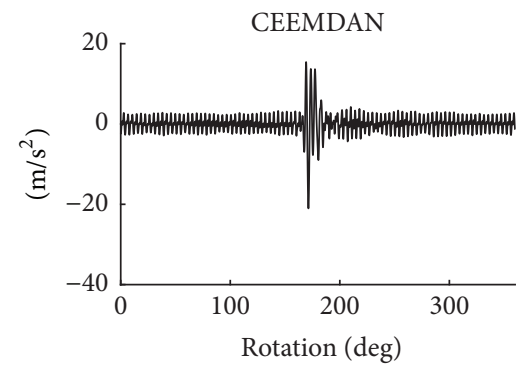

(c)

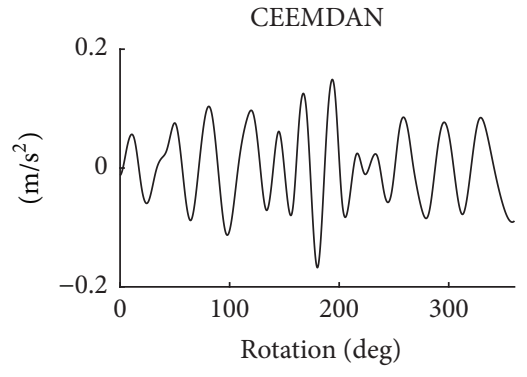

(f)

FIGURE 21: Representative signals of $(\mathrm{a}-\mathrm{c})$ gear II and (d-f) gear III for Case 2.

In order to test the reliability and the robustness of the methodology, simulated signals and two different real case studies have been analyzed by means of three different EMD algorithms. The first experimental case addresses a two-stage gearbox having an artificial gear tooth fault whereas the second one concerns a transmission system with a natural defect. The methodology successfully identifies the faulty gear in both the experimental tests, especially when the CEEMDAN is performed. On the basis of these results, the CEEMDAN is the most effective signal decomposition technique, since it returns the clearest results from both the qualitative and quantitative standpoints. Moreover, the proposed CIs-especially the NSVP-are very sensitive to the presence of a localized change of the vibration signature, simplifying the detection of the faulty gear.

On these grounds, the presented method can be considered reliable for the identification of a faulty gear when the fault occurs in a shaft with multiple gears. Eventually, this diagnosis method is particularly suitable for industrial applications since it is completely automatic. 
TABLE 5: CIs for the vibration signal of Case 2, with localized fault in gear II.

\begin{tabular}{|c|c|c|c|c|c|}
\hline & & Kurtosis & $\mathrm{CF}$ & $\mathrm{CPF}$ & NSVP \\
\hline \multirow{3}{*}{ EMD } & Gear II (faulty) & 48.803 & 10.910 & 13.704 & 0.085 \\
\hline & Gear III (healthy) & 12.182 & 4.979 & 4.317 & 0.089 \\
\hline & Gear II versus gear III [\%] & 300.604 & 119.140 & 217.457 & -5.017 \\
\hline \multirow{3}{*}{ EEMD } & Gear II (faulty) & 6.100 & 5.304 & 3.694 & 0.064 \\
\hline & Gear III (healthy) & 7.526 & 4.072 & 2.805 & 0.010 \\
\hline & Gear II versus gear III [\%] & -18.950 & 30.240 & 31.677 & 541.743 \\
\hline \multirow{3}{*}{ CEEMDAN } & Gear II (faulty) & 19.974 & 8.444 & 7.079 & 0.476 \\
\hline & Gear III (healthy) & 2.455 & 2.712 & 1.549 & 0.013 \\
\hline & Gear II versus gear III [\%] & 713.512 & 210.441 & 357.026 & 3696.969 \\
\hline
\end{tabular}

\section{Conflicts of Interest}

The authors declare that there are no conflicts of interest regarding the publication of this paper.

\section{References}

[1] R. B. Randall, Vibration-Based Condition Monitoring, John Wiley \& Sons Ltd, 2011.

[2] J. Antoni and R. B. Randall, "Differential diagnosis of gear and bearing faults," Journal of Vibration and Acoustics, vol. 124, no. 2, pp. 165-171, 2002.

[3] J. Antoni, "Cyclostationarity by examples," Mechanical Systems and Signal Processing, vol. 23, no. 4, pp. 987-1036, 2009.

[4] A. Raad, J. Antoni, and M. Sidahmed, "Indicators of cyclostationarity: theory and application to gear fault monitoring," Mechanical Systems and Signal Processing, vol. 22, no. 3, pp. 574587, 2008.

[5] T. Barszcz and R. B. Randall, "Application of spectral kurtosis for detection of a tooth crack in the planetary gear of a wind turbine," Mechanical Systems and Signal Processing, vol. 23, no. 4, pp. 1352-1365, 2009.

[6] G. Dalpiaz, A. Rivola, and R. Rubini, "Effectiveness and sensitivity of vibration processing techniques for local fault detection in gears," Mechanical Systems and Signal Processing, vol. 14, no. 3, pp. 387-412, 2000.

[7] G. L. McDonald, Q. Zhao, and M. J. Zuo, "Maximum correlated Kurtosis deconvolution and application on gear tooth chip fault detection," Mechanical Systems and Signal Processing, vol. 33, pp. 237-255, 2012.

[8] G. L. McDonald and Q. Zhao, "Multipoint Optimal Minimum Entropy Deconvolution and Convolution Fix: Application to vibration fault detection," Mechanical Systems and Signal Processing, vol. 82, pp. 461-477, 2017.

[9] V. Sharma and A. Parey, "Gear crack detection using modified TSA and proposed fault indicators for fluctuating speed conditions," Measurement, vol. 90, pp. 560-575, 2016.

[10] N. E. Huang, Z. Shen, S. R. Long et al., "The empirical mode decomposition and the Hilbert spectrum for nonlinear and non-stationary time series analysis," Proceedings A, vol. 454, no. 1971, pp. 903-995, 1998.

[11] Z. He, Y. Shen, and Q. Wang, "Boundary extension for HilbertHuang transform inspired by gray prediction model," Signal Processing, vol. 92, no. 3, pp. 685-697, 2012.

[12] X. Hu, S. Peng, and W.-L. Hwang, "EMD revisited: a new understanding of the envelope and resolving the mode-mixing problem in AM-FM signals," IEEE Transactions on Signal Processing, vol. 60, no. 3, pp. 1075-1086, 2012.

[13] N. E. Huang and Z. Wu, "Ensemble empirical mode decomposition: a noise-assisted data analysis method," Advances in Adaptive Data Analysis (AADA), vol. 1, no. 1, pp. 1-41, 2009.

[14] J.-R. Yeh, J.-S. Shieh, and N. E. Huang, "Complementary ensemble empirical mode decomposition: a novel noise enhanced data analysis method," Advances in Adaptive Data Analysis: Theory and Applications, vol. 2, no. 2, pp. 135-156, 2010.

[15] M. E. Torres, M. A. Colominas, G. Schlotthauer, and P. Flandrin, "A complete ensemble empirical mode decomposition with adaptive noise," in Proceedings of the 36th IEEE International Conference on Acoustics, Speech, and Signal Processing, pp. 41444147, Prague, Czech Republic, May 2011.

[16] S. J. Loutridis, "Damage detection in gear systems using empirical mode decomposition," Engineering Structures, vol. 26, no. 12, pp. 1833-1841, 2004.

[17] S. J. Loutridis, "Instantaneous energy density as a feature for gear fault detection," Mechanical Systems and Signal Processing, vol. 20, no. 5, pp. 1239-1253, 2006.

[18] J. Cheng, D. Yu, J. Tang, and Y. Yang, "Application of frequency family separation method based upon EMD and local Hilbert energy spectrum method to gear fault diagnosis," Mechanism and Machine Theory, vol. 43, no. 6, pp. 712-723, 2008.

[19] L. Hui, Y. Zhang, and H. Zheng, "Wear detection in gear system using Hilbert-Huang transform," Journal of Mechanical Science and Technology, vol. 20, no. 11, pp. 1781-1789, 2006.

[20] J. Lin and Q. Chen, "Application of the EEMD method to multiple faults diagnosis of gearbox," in Proceedings of the 2010 IEEE International Conference on Advanced Computer Control, ICACC 2010, pp. 395-399, March 2010.

[21] Z. P. Feng, M. Liang, Y. Zhang, and S. M. Hou, "Fault diagnosis for wind turbine planetary gearboxes via demodulation analysis based on ensemble empirical mode decomposition and energy separation," Journal of Renewable Energy, vol. 47, pp. 112-126, 2012.

[22] L. Zhao, W. Yu, and R. Yan, "Gearbox fault diagnosis using complementary ensemble empirical mode decomposition and permutation entropy," Shock and Vibration, vol. 2016, Article ID 3891429, 8 pages, 2016.

[23] Y. Lei, Z. Liu, J. Ouazri, and J. Lin, "A fault diagnosis method of rolling element bearings based on CEEMDAN," Proceedings of the Institution of Mechanical Engineers, Part C: Journal of Mechanical Engineering Science, vol. 231, no. 10, pp. 1804-1815, 2015.

[24] S. Mohanty, K. K. Gupta, and K. S. Raju, "Vibro-acoustic fault analysis of bearing using FFT, EMD, EEMD and CEEMDAN 
and their implications," Lecture Notes in Electrical Engineering, vol. 387, pp. 281-292, 2016.

[25] Y. Lei, J. Lin, Z. He, and M. J. Zuo, "A review on empirical mode decomposition in fault diagnosis of rotating machinery," Mechanical Systems and Signal Processing, vol. 35, no. 1-2, pp. 108-126, 2013.

[26] J. Antoni, F. Bonnardot, A. Raad, and M. El Badaoui, "Cyclostationary modelling of rotating machine vibration signals," Mechanical Systems and Signal Processing, vol. 18, no. 6, pp. 1285-1314, 2004.

[27] S. Braun, "The synchronous (time domain) average revisited," Mechanical Systems and Signal Processing, vol. 25, no. 4, pp. 1087-1102, 2011.

[28] G. Rilling and P. Flandrin, "One or two frequencies? The empirical mode decomposition answers," IEEE Transactions on Signal Processing, vol. 56, no. 1, pp. 85-95, 2008.

[29] P. Flandrin, G. Rilling, and P. Gonçalvés, "Empirical mode decomposition as a filter bank," IEEE Signal Processing Letters, vol. 11, no. 2, pp. 112-114, 2004.

[30] Z. H. Wu and N. E. Huang, "Ensemble empirical mode decomposition: a noise-assisted data analysis method," Advances in Adaptive Data Analysis (AADA), vol. 1, no. 1, pp. 1-41, 2009.

[31] Y. Lei, Z. He, and Y. Zi, "Application of the EEMD method to rotor fault diagnosis of rotating machinery," Mechanical Systems and Signal Processing, vol. 23, no. 4, pp. 1327-1338, 2009.

[32] M. A. Colominas, G. Schlotthauer, and M. E. Torres, "Improved complete ensemble EMD: a suitable tool for biomedical signal processing," Biomedical Signal Processing and Control, vol. 14, no. 1, pp. 19-29, 2014.

[33] M. A. Colominas, G. Schlotthauer, M. a. Torres, and P. Flandrin, "Noise-assisted EMD methods in action," Advances in Adaptive Data Analysis: Theory and Applications, vol. 4, no. 4, 1250025, 11 pages, 2012.

[34] R. B. Randall, "A new method of modeling gear faults," Journal of Mechanical Design, vol. 104, no. 2, pp. 259-267, 1982.

[35] J. Dybała and R. Zimroz, "Rolling bearing diagnosing method based on empirical mode decomposition of machine vibration signal," Applied Acoustics, vol. 77, pp. 195-203, 2014.

[36] B. Assaad, M. Eltabach, and J. Antoni, "Vibration based condition monitoring of a multistage epicyclic gearbox in lifting cranes," Mechanical Systems and Signal Processing, vol. 42, no. 1-2, pp. 351-367, 2014.

[37] W. Wang, "Early detection of gear tooth cracking using the resonance demodulation technique," Mechanical Systems and Signal Processing, vol. 15, no. 5, pp. 887-903, 2001.

[38] G. DElia, Fault detection in rotating machines by vibration signal processing techniques [PhD Thesis], Università degli Studi di Bologna, 2008.

[39] G. Dalpiaz, G. DElia, and S. Delvecchio, "Design of a test bench for the vibro-acoustical analysis and diagnostics of rotating machines," in in proceedings of The Second World Congress on Engineering Asset Management \& The Fourth International Conference on Condition Monitoring, pp. 777-792, Harrogate, UK, 2007. 


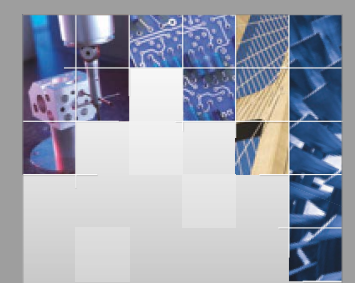

\section{Enfincering}
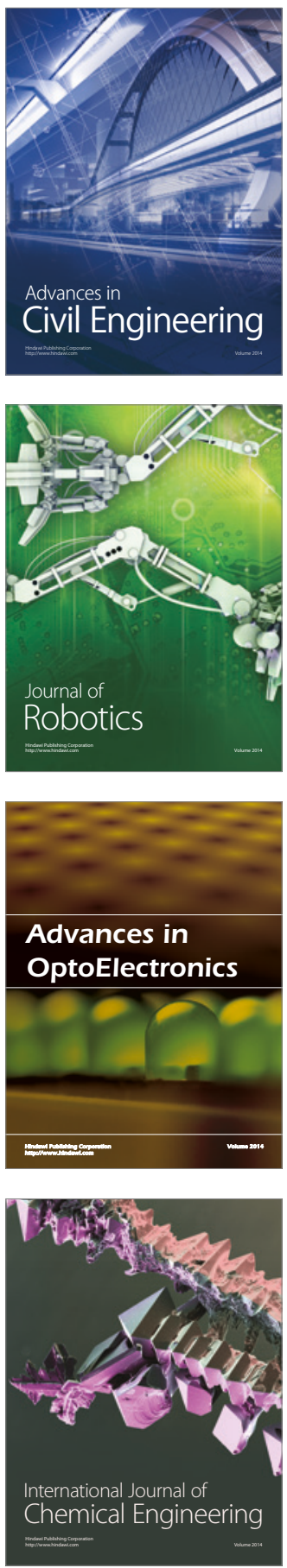

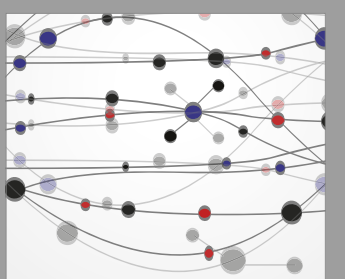

The Scientific World Journal

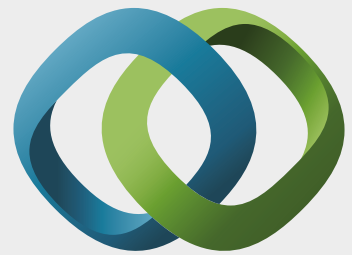

\section{Hindawi}

Submit your manuscripts at

https://www.hindawi.com
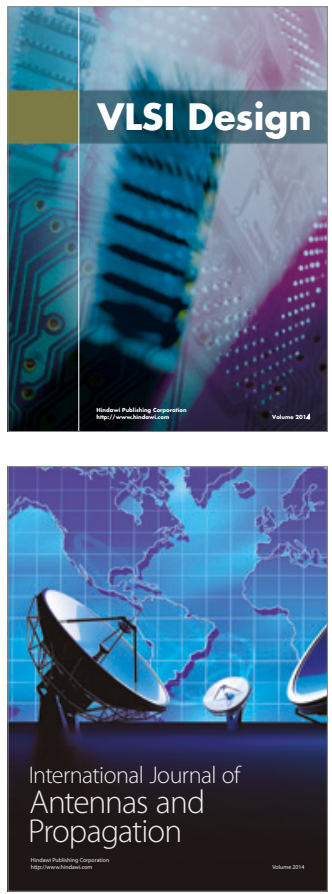

\section{Rotating}

Machinery
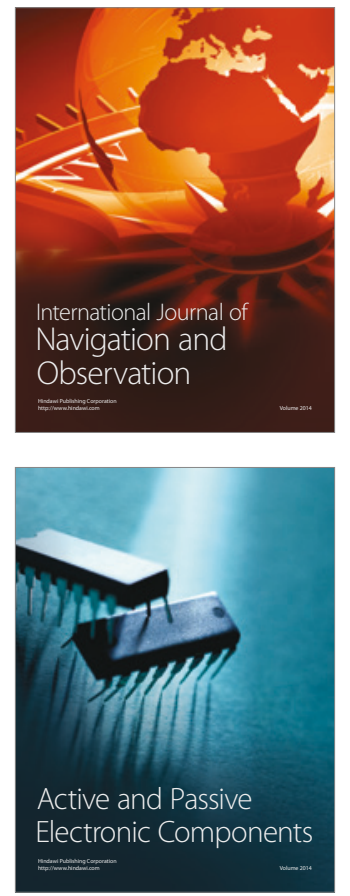
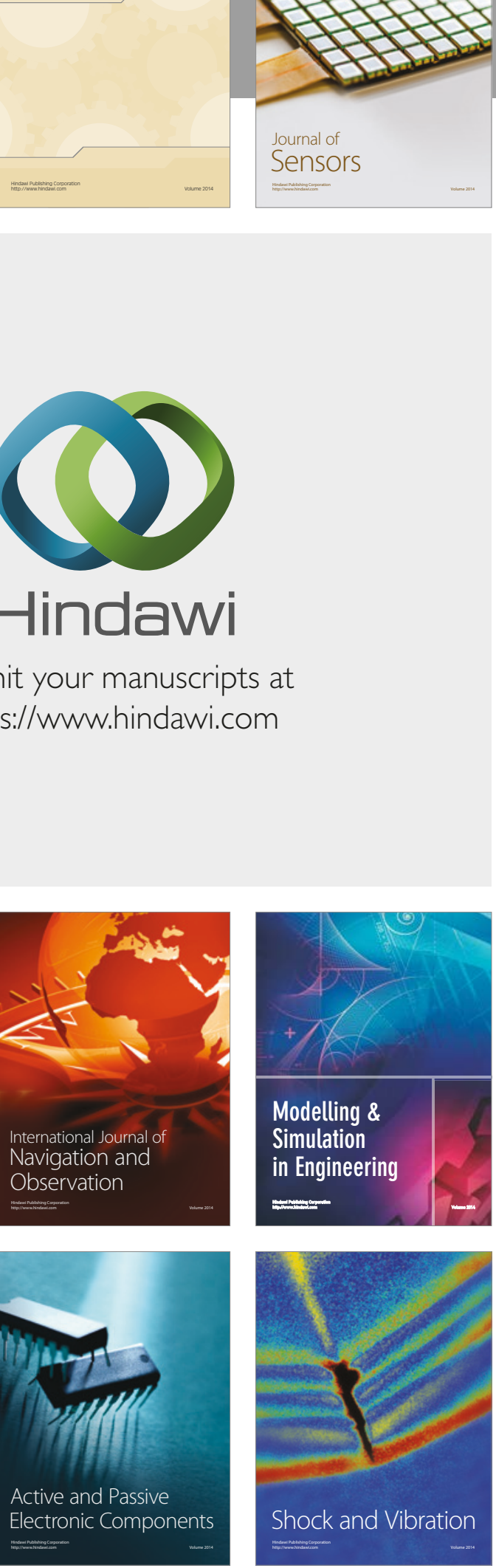
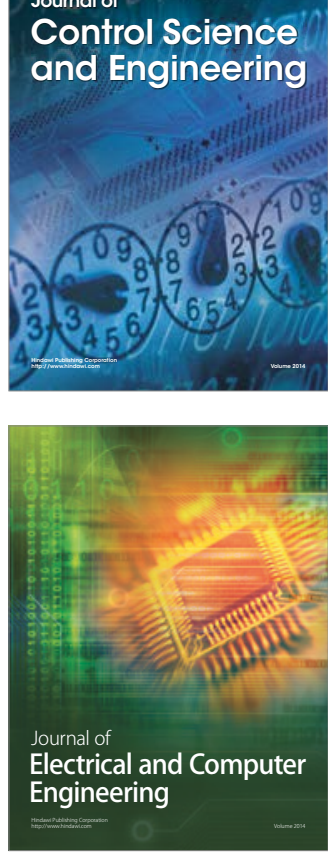

Distributed

Journal of

Control Science

and Engineering
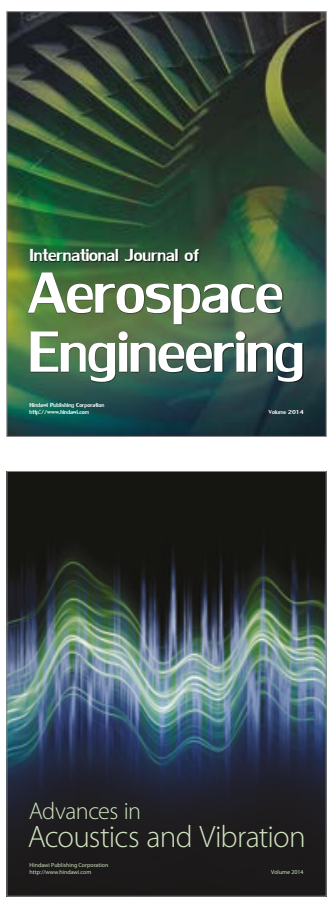

Sensor Networks 